\title{
On the Stochastic Limit of Quantum Field Theory
}

\author{
L. Accardi, J.Gough and Y.G. Lu
}

\begin{abstract}
The weak coupling limit for a quantum system, with discrete energy spectrum, coupled to a bose reservoir with the most general linear interaction is considered: under this limit we have a quantum noise processes substituting for the field. We obtain a limiting evolution unitary on the system and noise space which, when reduced to the sytem's degrees of freedom, provide the master and Langevin equations that are postulated on heuristic grounds by physicists. In addition we give a concrete application of our results by deriving the evolution of an atomic system interacting with the electrodynamic field without recourse to either rotating wave or dipole approximations.
\end{abstract}

\section{QUANTUM THEORY OF DAMPING}

trreversible quantum evolutions now play a fundamental role in many areas of physics especially quantum optics. A large body of physical literature has been built up around the problem of describing in a stochastic model the effect of a source of quantum noise on a given quantum mechanical system, emphasising the quantum stochastic properties of the source

of the quantum noise. However, quite generally, the classical approach to the stochasticity which is taken ignores the essential quantum nature of the problem. In this paper we wish to develop the weak coupling approach [1 to quantum damping. In a sense the noise feilds used to model physical sources are still quantum in nature, as will be explained later, and because they are arrive at from a well defined physical scaling limit do not require us to put in the desired features of the noise by hand.

The derivations of the quantum master equations and quantum Langevin equations for open quantum systems which are present in the current physics literature are well motivated from the physical point of view, cf. [2], however mathematically imprecise. The heuristic procedure to render the reservoir, to which the system is coupled, into a source of quantum noise via some markovian approximation is generally ill-defined and reliant on second order perturbation theory.

On the other hand, the weak coupling limit for an open quantum system gives a device for obtaining irreversible evolutions. $\checkmark$ Mathematically rigorous derivations of the Langevin and master equations along these lines have been given for certain specific models by Pulé [3] and Davies [4]. Other attempts have been made to approximate a quantum reservoir by fitting Quantum Brownian Motions (QBMs), cf. [5], [6] and [7.

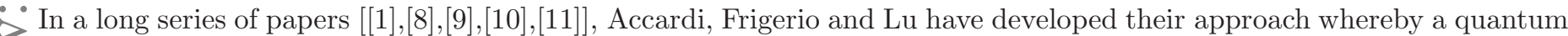
reservoir can be reduced to a quantum stochastic noise source via a weak coupling limit procedure. The theory is mathematically rigorous while at the same time applicable to the wide range of phenomena considered by physicists and gives a precise description of the reservoir as a quantum noise source. The main mathematical device for establishing convergence of the reservoir feilds is a quantum central limit theorem; that is a central limit theorem for non-commuting observables: the theory of non-commutative probability, or Quantum Probability as it is more correctly known, affords the necessary mathematical framework to interpret the limit process. Indeed the Fock space descriptions of a bose reservoir and a quantum stochastic processes are so similar in nature, it is not surprising, with hindsight, that the former may be reduced to the latter following some limiting procedure.

Our objective in this paper is to review the results of the programme of Accardi, Frigerio and Lu so far and to extend their theory so as to deal with the most general interaction between a system and a noise source encountered in physical theories. This we do and show that the energy shifts, linewidths, master equations and Langevin equations, arising for the system as a result of its coupling to the noise field, concur with those obtain by earlier researchers 2]. However we have, in addition, also a quantum stochastic description of the reservoir noise fields themselves. As a concrete application of our theory we consider the particular case where a quantum electrodynamical field acts as reservoir, however we stress that this is only one of the many applications of our theory. 
We shall discuss only minimal coupling interacions, that is interactions linear in the creation/ annihilation operators for the reservoir. In a later paper we discuss how to treat the situations where the interaction is of polynomial type.

\section{$1.1 \quad$ Open Quantum Systems}

We consider a system (S) coupled to a reservoir (R). The system $(\mathrm{S})$ is to be a quantum mechanical: its state space shall be a separable Hilbert space $\mathcal{H}_{S}$. The reservoir, on the other hand, is comprised of one or indeed several quantum fields, and so has infinitely many degrees of freedom. We shall consider a bosonic reservoir; the state space for $(R)$ is the bosonic Fock space $\mathcal{H}_{R}$ over a separable Hilbert space $\mathcal{H}_{R}^{1}$; in standard notation we write $\mathcal{H}_{R}=\Gamma_{B}\left(\mathcal{H}_{R}^{1}\right)$. $\mathcal{H}_{R}^{1}$ is again to be a separable Hilbert space and may quite generally describe not only one but several individual species of particle in the reservoir. For instance, consider several species of particle $P_{1}, P_{2}, P_{3}, \ldots$ in the reservoir and suppose that $\mathcal{H}_{R}^{1}=\oplus_{j} \mathcal{H}_{P_{j}}^{1}$, where $\mathcal{H}_{P_{j}}^{1}$ is the state space for particle type $P_{j}$, then

$$
\mathcal{H}_{R}=\Gamma_{B}\left(\mathcal{H}_{R}^{1}\right)=\Gamma_{B}\left(\oplus_{j} \mathcal{H}_{P_{j}}^{1}\right)=\otimes_{j} \Gamma_{B}\left(\mathcal{H}_{P_{j}}^{1}\right) .
$$

The space $\mathcal{H}_{R}^{1}$ is referred to as the (combined) one particle state space for the reservoir. The overall state space for the combined system and reservoir is $\mathcal{H}_{S} \otimes \mathcal{H}_{R}$. The vacuum vector of the reservoir space will be denoted throughout as $\Psi_{R}$. In the following we consider only bosonic species in the reservoir, however it is also possible to work with fermions 9 . The dynamics of the combined system and reservoir is governed by the formal Hamiltonian $H^{(\lambda)}$ which we may write as

$$
H^{(\lambda)}=H^{(0)}+\lambda H_{I}
$$

that is, as the sum of a free Hamiltonian $H^{(0)}$ and an interaction $H_{I}$, with $\lambda$ a real coupling parameter. $H^{(0)}$ is to be expressible as

$$
H^{(0)}=H_{S} \otimes 1_{R}+1_{S} \otimes H_{R},
$$

where $H_{S}$ and $H_{R}$ are self-adjoint operators on the spaces $\mathcal{H}_{S}$ and $\mathcal{H}_{R}$ respectively. For each $\lambda$, we consider the unitary operator $V_{t}^{(\lambda)}$ on $\mathcal{H}_{S} \otimes \mathcal{H}_{R}$ defined by

$$
V_{t}^{(\lambda)}=\exp \frac{t}{i \hbar} H^{(\lambda)}
$$

This gives the time evolution under $H^{(\lambda)}$. A standard device in pertubation theory is to transform to the interaction picture; this involves introducing the operator

$$
U_{t}^{(\lambda)}=V_{t}^{(0) \dagger} V_{t}^{(\lambda)} .
$$

$U_{t}^{(\lambda)}$ is a unitary operator on $\mathcal{H}_{S} \otimes \mathcal{H}_{R}$ called the wave operator at time $t$. We note that $\left\{U_{t}^{(\lambda)}: t \in \mathbb{R}\right\}$ is a left $v_{t}^{(0)}$-cocycle, that is it satisfies the relation

$$
U_{t+s}^{(\lambda)}=v_{t}^{(0)}\left(U_{s}^{(\lambda)}\right) U_{t}^{(\lambda)} .
$$

We define the time-evolutes for $X \in \mathcal{B}\left(\mathcal{H}_{S}\right) \otimes \mathcal{B}\left(\mathcal{H}_{R}\right)$ as

$$
\begin{aligned}
& v_{t}^{(\lambda)}(X)=V_{t}^{(\lambda) \dagger} X V_{t}^{(\lambda)}, \\
& u_{t}^{(\lambda)}(X)=U_{t}^{(\lambda) \dagger} X U_{t}^{(\lambda)} .
\end{aligned}
$$

The Schrödinger equation for the time evolutions are

$$
\frac{\partial}{\partial t} V_{t}^{(\lambda)}=\frac{1}{i \hbar} H^{(\lambda)} V_{t}^{(\lambda)}
$$

and

$$
\frac{\partial}{\partial t} U_{t}^{(\lambda)}=\frac{\lambda}{i \hbar} v_{t}^{(0)}\left(H_{I}\right) U_{t}^{(\lambda)} .
$$

With these we deduce the associated Heisenberg equations

$$
\frac{\partial}{\partial t} v_{t}^{(\lambda)}(X)=\frac{1}{i \hbar}\left[v_{t}^{(\lambda)}(X), v_{t}^{(\lambda)}\left(H^{(\lambda)}\right)\right]=\frac{1}{i \hbar} v_{t}^{(\lambda)}\left(\left[X, H^{(\lambda)}\right]\right)
$$

and

$$
\frac{\partial}{\partial t} u_{t}^{(\lambda)}(X)=\frac{\lambda}{i \hbar} u_{t}^{(\lambda)}\left(\left[X, v_{t}^{(0)}\left(H_{I}\right)\right]\right)
$$


From (8) we obtain the integral equation

$$
U_{t}^{(\lambda)}=1+\frac{\lambda}{i \hbar} \int_{0}^{t} d s v_{s}^{(0)}\left(H_{I}\right) U_{s}^{(\lambda)}
$$

and consequently the iterated Born series

$$
U_{t}^{(\lambda)}=1+\sum_{n=1}^{\infty}\left(\frac{\lambda}{i \hbar}^{n} \int_{0}^{t} d t_{1} \ldots \int_{0}^{t_{n-1}} d t_{n} v_{t_{1}}^{(0)}\left(H_{I}\right) \ldots v_{t_{n}}^{(0)}\left(H_{I}\right) .\right.
$$

This may be resummed as

$$
U_{t}^{(\lambda)}=\mathcal{T} \exp \left\{\frac{\lambda}{i \hbar} \int_{0}^{t} d s v_{s}^{(0)}\left(H_{I}\right)\right\}
$$

where $\mathcal{T}$ denotes time ordering.

Before we continue, we must say more about how to interpret the formal sum of $H^{(0)}$ and $H_{I}$. Firstly we assume that $H^{(0)}$ and $H_{I}$ are self-adjoint operators on $\mathcal{H}_{S} \otimes \mathcal{H}_{R}$. We shall assume that, for sufficiently small $\lambda$ and bounded $t$, the iterated series (11) is uniformly convergent and is bounded on $\mathcal{H}_{S} \underline{\otimes} \mathcal{E}\left(\mathcal{H}_{R}^{1}\right)$, the algebraic tensor product of $\mathcal{H}_{S}$ nd the set of exponential vectors. From the cocycle relation (1.1:6) we have that if we define the unitary operator $V_{t}^{(\lambda)}$ by

$$
V_{t}^{(\lambda)}=V_{t}^{(0)} U_{t}^{(\lambda)}
$$

then $\left\{V_{t}^{(\lambda)}: t \in \mathbb{R}\right\}$ gives a strongly continuous unitary group whose generator $\frac{1}{\hbar} H^{(\lambda)}$ is formally given as $H^{(\lambda)}=$ $H^{(0)}+\lambda H_{I}$. The time evolution in the Heisenberg picture is then given by

$$
v_{t}^{(\lambda)}(X)=u_{t}^{(\lambda)}\left(v_{t}^{(0)}(X)\right) .
$$

\subsection{The Free Evolution}

For $\mathcal{H}_{R}^{1}=L^{2}\left(\mathbb{R}^{3}\right)$ (momentum space), we have creation/ annihilation operators $a^{\sharp}(\mathbf{k})$ satisfying

$$
\left[a(\mathbf{k}), a\left(\mathbf{k}^{\prime}\right)^{\dagger}\right]=\delta\left(\mathbf{k}-\mathbf{k}^{\prime}\right),\left[a(\mathbf{k})^{\dagger}, a^{\dagger}\left(\mathbf{k}^{\prime}\right)\right]=0,\left[a(\mathbf{k}), a\left(\mathbf{k}^{\prime}\right)\right]=0 .
$$

The creation/ annihilation fields on $\mathcal{H}_{R}$ are defined, for $g \in \mathcal{H}_{R}^{1}$, by

$$
A^{\dagger}(g)=\int d^{3} k g(\mathbf{k}) a^{\dagger}(\mathbf{k}), A(g)=\int d^{3} k \bar{g}(\mathbf{k}) a(\mathbf{k}) .
$$

From (16) we obtain the canonical commutation relations (CCR):

$\left[A(h), A^{\dagger}(f)\right]=\langle h, f\rangle$, while $[A(h), A(f)]=0$. We take $H_{R}$ to be the second quantization of an operator $H_{R}^{1}$ on $\mathcal{H}_{R}^{1}$ given by

$$
\left(H_{R}^{1} f\right)(k)=\hbar \omega(k) f(k) .
$$

$H_{R}$ may then be expressed as

$$
H_{R}=\int d k \hbar \omega(k) a^{\dagger}(k) a(k)
$$

Note that $v_{t}^{(0)}\left(1_{S} \otimes A^{\sharp}(g)\right)=1_{S} \otimes A^{\sharp}\left(S_{t} g\right)$; where we have introduced the unitary operator $S_{t}$ on $\mathcal{H}_{R}^{1}$ given by

$$
S_{t}=\exp \left\{-\frac{t}{i \hbar} H_{R}^{1}\right\}
$$

that is $\left(S_{t} f\right)(k)=e^{i \omega(k) t} f(k)$. 


\subsection{The standard approach to the quantum Langevin equation}

Consider an interaction of the type

$$
H_{I}=i \hbar\left\{D \otimes A^{\dagger}(g)-D^{\dagger} \otimes A(g)\right\}
$$

where $D \in \mathcal{B}\left(\mathcal{H}_{S}\right)$ has a harmonic free-evolution; that is $\frac{1}{i \hbar}\left[D, H_{S}\right]=-i \omega D$. Then we have

$$
v_{t}^{(0)}\left(H_{I}\right)=i \hbar\left\{D \otimes A^{\dagger}\left(S_{t}^{\omega} g\right)-D^{\dagger} \otimes A\left(S_{t}^{\omega} g\right)\right\}
$$

where

$$
S_{t}^{\omega}=e^{-i \omega t} S_{t}
$$

that is, $\left(S_{t}^{\omega} f\right)(\mathbf{k})=e^{i(\omega(\mathbf{k})-\omega) t} f(\mathbf{k})$. Now write $X_{t}=u_{t}^{(\lambda)}\left(X \otimes 1_{R}\right)$, for $X \in \mathcal{B}\left(\mathcal{H}_{S}\right)$, then from (9) we have

$$
\frac{\partial X_{t}}{\partial t}=\lambda\left\{A_{t}^{\dagger}\left(S_{t}^{\omega} g\right)[X, D]_{t}-\left[X, D^{\dagger}\right]_{t} A_{t}\left(S_{t}^{\omega} g\right)\right\}
$$

where $A_{t}^{\sharp}(f)=u_{t}^{(\lambda)}\left(1_{S} \otimes A^{\sharp}(f)\right)$.

Now one can show that $A_{t}(f)=\lambda \int_{0}^{\infty} d s D_{s}\left\langle f, S_{s}^{\omega} g\right\rangle+1_{S} \otimes A(f)$, so substituting back into (23) gives

$$
\frac{\partial X_{t}}{\partial t}=\lambda^{2} \int_{0}^{\infty}\left\{D_{s}^{\dagger} \phi^{\omega}(s-t)[X, D]_{t}-\left[X, D^{\dagger}\right]_{t} \phi^{\omega}(t-s) D_{s}\right\}+\lambda\left\{\xi_{t}^{\omega}[X, D]_{t}-\left[X, D^{\dagger}\right]_{t} \xi_{t}^{\omega \dagger}\right\}
$$

where $\phi^{\omega}(t)=\left\langle g, S_{-t}^{\omega} g\right\rangle=\int d^{3} k|g(\mathbf{k})|^{2} e^{-i(\omega(\mathbf{k})-\omega) t}$ and $\xi^{\omega}(t)=1_{S} \otimes A\left(S_{t}^{\omega} g\right)$. In standard terminology $\phi(t)$ is called the memory function and $\xi_{t}^{\omega}$ the fluctuating quantum force $[2,17]$ or input field [18], albeit in the interaction picture. One notes that, in the vacuum state, $\xi_{t}^{\omega}$ is gaussian distributed and all first and second moments vanish except the two-point function

$$
\left\langle\Psi_{R}, \xi_{t}^{\omega} \xi_{t}^{\omega \dagger} \Psi_{R}\right\rangle=\left\langle S_{t}^{\omega} g, S_{s}^{\omega} g\right\rangle=\phi^{\omega}(t-s) .
$$

The standard approach taken at this juncture is to introduce the so-called first Markov approximation. Here, for example, one takes $\mathcal{H}_{R}^{1}=L^{2}(\mathbb{R}), g=\sqrt{\frac{\kappa}{2 \pi}}$ (constant) and $\omega(k)=k$. Then

$$
\phi^{\omega}(t)=\int_{-\infty}^{\infty} d k \frac{\kappa}{2 \pi} e^{i(k+\omega) t}=\kappa e^{i \omega t} \delta(t) .
$$

There are, however, several important objections to be made to this approach. Firstly any physical details specific to the reservoir must be put in by hand. Secondly, the spectrum of $H_{R}^{1}$ is here unbounded below, this is necessary to produce the delta function correlation of white noise. From a physical point of view this is unacceptable as $H_{R}^{1}$ must be bounded below for stability. Finally, the fact that the frequency spectrum $\omega(k)=k$ is unbounded below precludes any possibility of dropping the rotating wave approximation.

\subsection{The Weak Coupling Limit}

We now describe the ideas behind the weak coupling limit in the simplest situation where we have taken a dipole and rotating wave approximation. We define the following reservoir operator

$$
B_{t}^{(\omega, \lambda)}(g)=A\left(\lambda \int_{0}^{t} d t_{1} S_{t_{1}}^{\omega} g\right)=\lambda \int_{M} \mu(d k) \int_{0}^{t} d t_{1} e^{-i(\omega(k)-\omega) t_{1}} \bar{g}(k) a(k) .
$$

Calculating the two-point vacuum expectations gives

$$
\begin{gathered}
\left\langle\Psi_{R}(0), B_{t}^{(\omega, \lambda)}(g) B_{s}^{(\omega, \lambda) \dagger}(f) \Psi_{R}(0)\right\rangle=\lambda^{2} \int_{0}^{t} d t_{1} \int_{0}^{s} d s_{1}\left\langle S_{t_{1}}^{\omega} g, S_{s_{1}}^{\omega} f\right\rangle \\
\equiv \int_{0}^{\lambda^{2} t} d u \int_{u / \lambda^{2}-s}^{u / \lambda^{2}} d \tau\left\langle S_{\tau}^{\omega} g, f\right\rangle
\end{gathered}
$$

where we have substituted $u=\lambda^{2} t_{1}$ and $\tau=t_{1}-s_{1}$. 
In order to obtain a non-trivial two-point function in the limit $\lambda \rightarrow 0$ we must rescale time as

$$
t \hookrightarrow t / \lambda^{2}
$$

this is known as the van Hove or weak coupling limit in physics. One finds

$$
\lim _{\lambda \rightarrow 0}\left\langle\Psi_{R}, B_{t / \lambda^{2}}^{(\omega, \lambda)}(g) B_{s / \lambda^{2}}^{(\omega, \lambda) \dagger}(f) \Psi_{R}\right\rangle=\min (t, s) \int_{-\infty}^{\infty} d \tau\left\langle S_{\tau}^{\omega} g, f\right\rangle .
$$

Physically the limit $\lambda \rightarrow 0$ with $t \hookrightarrow t / \lambda^{2}$ allows us to consider progressively weaker interactions which are allowed to run over increasingly larger periods of time and so we obtain the long term cumulative effect of the interaction on the system. Now the creation and annihilation operators are gaussian in the vaccum state and, as a result, so too are the operators $B_{t / \lambda^{2}}^{(\omega, \lambda) \sharp}$.

Furthermore the limiting two-point function (29) is suggestive of the correlation function of a Brownian motion. However, an interpretation of the above in terms of classical Brownian motion is erroneous as it ignores the essentially quantum probabilistic nature of these processes.

\subsection{The Interaction}

For technical reasons we work with a system Hamiltonian $H_{S}$ which has discrete spectrum. $H_{R}^{1}$ is taken to be bounded below as required from physics. The type of interaction $H_{I}$ which we wish to study is of the form

$$
H_{I}=i \hbar \sum_{\omega \in F} \sum_{j=1}^{N(\omega)}\left\{D_{j}^{\omega} \otimes A^{\dagger}\left(g_{j}^{\omega}\right)-D_{j}^{\omega \dagger} \otimes A\left(g_{j}^{\omega}\right)\right\},
$$

where $F$ is a discrete subset of $\mathbb{R}$. For each $\omega \in F$, we take $D_{j}^{\omega} \in \mathcal{B}\left(\mathcal{H}_{S}\right)$ to have harmonic free evolution with frequency $\omega:$

$$
\frac{1}{i \hbar}\left[D_{j}^{\omega}, H_{S}\right]=-i \omega D_{j}^{\omega}, j=1, \ldots, N(\omega) .
$$

Thus the superscript $\omega$ labels harmonic frequency and $j=1, \ldots, N(\omega)$ the degeneracy of that frequency. An interaction similar to (30) has been treated in [10], however there the test functions $g_{j}^{\omega}$ were taken to be equal for each value of $\omega$. Our reasons for studying (30) above are because it allows us to treat the most general interactions encountered in physics. Typically in quantum field theory one considers an interaction of the type

$$
H_{I}=i h \int d^{3} k\left\{\theta(\mathbf{k}) \otimes a^{\dagger}(\mathbf{k})-\theta^{\dagger}(\mathbf{k}) \otimes a(\mathbf{k})\right\}
$$

where $\left\{\theta(\mathbf{k}): \mathbf{k} \in \mathbb{R}^{3}\right\}$ is a family of operators on $\mathcal{H}_{S}$. The operators $\theta(\mathbf{k})$ are called the response terms: they contain local information about the interaction. In the dipole approximation of quantum field theory one makes the replacement

$$
\theta(\mathbf{k}) \hookrightarrow \theta^{\text {dipole }}(\mathbf{k})=g(\mathbf{k}) \theta(0),
$$

where $g(\mathbf{k})$ is some suitable test function. The physical argument is, cf. [2, that the response does not vary appreciably for values of the wavelength of the reservoir particles which are large relative to the physical dimensions of the system, though this can hardly be true for large momenta. As a result one obtains the approximate Hamiltonian

$$
H_{I} \hookrightarrow H_{I}^{\text {dipole }}=i \hbar\left\{\theta(0) \otimes A^{\dagger}(g)-\theta^{\dagger}(0) \otimes A(g)\right\} .
$$

A further approximation often made by physicists is to replace $\theta(0)$ by an operator $D$ having a harmonic free-evolution with some frequency $\omega \in \mathbb{R}$. This approximation is just the rotating wave approximation.

In order to avoid these appproximations we argue as follows: Let $B$ be a complete basis of eigenstates of $H_{S}$, then

$$
H_{I}=\sum_{\phi, \phi^{\prime} \in B}\left\langle\phi\left|H_{I}\right| \phi^{\prime}\right\rangle|\phi\rangle\left\langle\phi^{\prime}\right|,
$$

but however we may write

$$
\left\langle\phi\left|H_{I}\right| \phi^{\prime}\right\rangle=i \hbar \int d k\left\{\left\langle\phi|\theta(\mathbf{k})| \phi^{\prime}\right\rangle a^{\dagger}(\mathbf{k})-\left\langle\phi\left|\theta^{\dagger}(\mathbf{k})\right| \phi^{\prime}\right\rangle a(\mathbf{k})\right\}
$$




$$
\equiv i \hbar\left[A^{\dagger}\left(g_{\phi \phi^{\prime}}\right)-A\left(g_{\phi^{\prime} \phi}\right)\right]
$$

where we have introduced the test functions

$$
g_{\phi \phi^{\prime}}(\mathbf{k})=\left\langle\phi|\theta(\mathbf{k})| \phi^{\prime}\right\rangle:
$$

note that the order of $\phi$ and $\phi^{\prime}$ is reversed in the second term in due to the conjugate linear nature of the creation feild. This now means that the interaction can be expressed as

$$
H_{I}=i \hbar \sum_{\phi, \phi^{\prime} \in B}\left\{T_{\phi \phi^{\prime}} \otimes A^{\dagger}\left(g_{\phi \phi^{\prime}}\right)-T_{\phi \phi^{\prime}}^{\dagger} \otimes A\left(g_{\phi \phi^{\prime}}\right)\right\},
$$

where we have introduced the transition operators $T_{\phi \phi^{\prime}}=|\phi\rangle\left\langle\phi^{\prime}\right|$.

We note that the transition operators $T_{\phi \phi^{\prime}}$ are harmonic under the free evolution, in fact we have

$$
\frac{1}{i \hbar}\left[T_{\phi \phi^{\prime}}, H_{S}\right]=-i \omega_{\phi \phi^{\prime}} T_{\phi \phi^{\prime}},
$$

where

$$
\omega_{\phi \phi^{\prime}}=\frac{E_{\phi^{\prime}}-E_{\phi}}{\hbar} .
$$

So $F=\left\{\omega_{\phi \phi^{\prime}}: \phi, \phi^{\prime} \in B\right\}$ is now the set of Bohr frequencies.

The expression (38) is now equivalent to the interaction (30) which we propose to study. Here we need only relabel the $T_{\phi \phi^{\prime}}$ as $D_{j}^{\omega}$ where $\omega=\omega_{\phi \phi^{\prime}}$ and the $j$ again labels degeneracy. The functions $g_{\phi \phi^{\prime}}$ are relabeled accordingly.

\section{THE QUANTUM STOCHASTIC LIMIT}

\subsection{Quantum Brownian Motions}

In this section we first of all discuss the concept of quantum brownian motion. As this is not yet widely known amongst physicists we give an exposition below:

Definition. A quantum brownian motion $(Q B M)$ is a triple $\left(H, \Phi,\left(B_{t}\right)_{t}\right)$, where $H$ is a separable Hilbert space, $\Phi \in H$ with $\|\Phi\|=1,\left(B_{t}\right)_{t}$ is a family of operators on $H$ such that

(i) $q_{t}=\operatorname{Re} B_{t}$ and $p_{t}=\operatorname{Im} B_{t}$ are classical brownian motions for the state $\Phi$.

(ii) $\left[p_{s}, q_{t}\right]=\frac{\kappa}{2 i} \min (s, t)$, where $\kappa \in R$.

The basic example is the following: Let $\mathcal{H}_{\mathbb{C}}=\Gamma_{B}\left(L^{2}(\mathbb{R})\right)$ and $\Phi_{\mathbb{C}}$ be the vacuum state. Then define $B_{t}$ to be

$$
B_{t}=A_{\mathbb{C}}\left(\chi_{[0, t]}\right)
$$

where $A_{\mathbb{C}}$ is the annihilation operator on $\mathcal{H}_{\mathbb{C}}$. From the $(\mathrm{CCR})$ we have

$$
\left[B_{t}, B_{s}^{\dagger}\right]=\left\langle\chi_{[0, t]}, \chi_{[0, s]}\right\rangle=\min (t, s) ;\left[B_{t}, B_{s}\right]=0=\left[B_{t}^{\dagger}, B_{s}^{\dagger}\right]
$$

So setting $q_{t}=\frac{1}{2}\left(B_{t}+B_{t}^{\dagger}\right)$ and $p_{t}=\frac{1}{2 i}\left(B_{t}-B_{t}^{\dagger}\right)$ we have from the $(\mathrm{CCR})$ that $\left[p_{t}, q_{s}\right]=-\left[q_{t}, p_{s}\right]$ and

$$
\left[p_{t}, q_{s}\right]=\frac{1}{2 i} \min (t, s) .
$$

Now if we set

$$
d B_{t}=B_{t+d t}-B_{t}=A_{\mathbb{C}}\left(\chi_{[t, t+d t]}\right),
$$

we have that

$$
\begin{gathered}
\left\langle\Phi_{\mathbb{C}}, d B_{t}^{\sharp} \Phi_{\mathbb{C}}\right\rangle=0 ;\left\langle\Phi_{\mathbb{C}},\left(d B_{t}^{\sharp}\right)^{2} \Phi_{\mathbb{C}}\right\rangle=0 ;\left\langle\Phi_{\mathbb{C}}, d B_{t}^{\dagger} d B_{t} \Phi_{\mathbb{C}}\right\rangle=0 ; \\
\left\langle\Phi_{\mathbb{C}}, d B_{t} d B_{t}^{\dagger} \Phi_{\mathbb{C}}\right\rangle=d t .
\end{gathered}
$$

Now $d B_{t}$ and $d B_{t}^{\dagger}$ are gaussian in the vacuum state, because the creation and annihilation fields are, therefore so too are $d q_{t}$ and $d p_{t}$. Furthermore,

$$
\left\langle\Phi_{\mathbb{C}},\left(d q_{t}\right)^{2} \Phi_{\mathbb{C}}\right\rangle=\frac{1}{4}\left\langle\Phi_{\mathbb{C}},\left(d B_{t}+d B_{t}^{\dagger}\right)^{2} \Phi_{C}\right\rangle=\frac{1}{4} d t
$$


and similarly $\left\langle\Phi_{\mathbb{C}},\left(d p_{t}\right)^{2} \Phi_{C}\right\rangle=\frac{1}{4} d t$. Finally noting that at unequal times $s$ and $t$

$$
\left\langle\Phi_{\mathbb{C}}, d q_{t} d q_{s} \Phi_{\mathbb{C}}\right\rangle=0=\left\langle\Phi_{\mathbb{C}}, d p_{t} d p_{s} \Phi_{\mathbb{C}}\right\rangle
$$

whenever $t\left\langle t+d t \leq s\left\langle s+d s\right.\right.$ or $s\left\langle s+d s \leq t\left\langle t+d t\right.\right.$, we conclude that $\left(q_{t}\right)_{t}$ and $\left(p_{t}\right)_{t}$ are each separate Brownian motions for expectations taken in the state $\Phi_{\mathbb{C}}$. So $\left\{\mathcal{H}_{\mathbb{C}}, \Phi_{\mathbb{C}},\left(B_{t}\right)_{t}\right\}$ is a QBM. We can introduce formal creation and annihilation densities $b^{\sharp}(t)$ satisfying

$$
[b(t), b(s)]=0=\left[b^{+}(t), b^{+}(s)\right] ;\left[b(t), b^{+}(s)\right]=\delta(t-s),
$$

such that

$$
A_{\mathbb{C}}(g)=\int_{\mathbb{R}} d s \overline{g(s)} b(s) ; A_{\mathbb{C}}^{\dagger}(g)=\int_{\mathbb{R}} d s g(s) b^{+}(s)
$$

from this we see

$$
B_{t}^{\sharp}=\int_{0}^{t} d s b^{\sharp}(s) .
$$

We may write $b_{t}^{\sharp}=\frac{d B_{t}}{d t}$ and consider these densities as "quantum white noises".

More generally let $K$ be a separable Hilbert space and let $L^{2}(\mathbb{R}, K)$ denote the set of square-integrable $K$-valued functions over $\mathbb{R}$. Now $h \in L^{2}(\mathbb{R}, K)$ is a function $h(t) \in K$ with $\int_{\mathbb{R}} d t\|h(t)\|_{K}^{2}\left\langle\infty\right.$. The inner product on $L^{2}(\mathbb{R}, K)$ is given by

$$
\left\langle h, h^{\prime}\right\rangle=\int_{\mathbb{R}}\left\langle h(t), h^{\prime}(t)\right\rangle d t
$$

If $\left\{e_{n}\right\}_{n}$ is a complete orthonormal basis for $K$ then we can write $h(t)=\sum_{n} h_{n}(t) e_{n}$, where $h_{n}(t)=\left\langle e_{n}, h(t)\right\rangle_{K}$; this gives a natural isomorphism

$$
L^{2}(\mathbb{R}, K) \cong K \otimes L^{2}(\mathbb{R}) .
$$

Now take $\mathcal{H}_{K}=\Gamma_{B}\left(L^{2}(\mathbb{R}, K)\right)$ and let $\Phi_{K}$ denote vacuum vector of $\mathcal{H}_{K}$, then a QBM is given by $\left(\mathcal{H}_{K}, \Phi_{K},\left(B_{t}(g)\right)_{t}\right)$, for non-zero $g \in K$, where

$$
B_{t}(g)=A_{K}\left(g \otimes \chi_{[0, t]}\right)
$$

where $A_{K}$ is the annihilation operator on $\mathcal{H}_{K}$. The commutation relations are

$$
\left[B_{t}(g), B_{s}^{\dagger}(f)\right]=\left\langle g \otimes \chi_{[0, t]}, f \otimes \chi_{[0, s]}\right\rangle=\langle g, f\rangle_{K} \min (t, s)
$$

with remaining commutators vanishing. So $\left\{\mathcal{H}_{K}=\Gamma_{B}\left(L^{2}(\mathbb{R}, K)\right), \Phi_{K},\left(B_{t}(g)\right)_{t}\right\}$ is a QBM. Taking $K=\mathbb{C}$ and $|g|^{2}=\kappa$ leads back to the original example.

However, there is a more general possibility than that above; Let $Q \geq 1_{K}$ and set

$$
C=Q \otimes 1_{L^{2}(\mathbb{R})}
$$

Then let $\varphi_{C}$ be the state on the Weyl algebra $W\left(\mathcal{H}_{K}\right)$ with covariance $C$. We can construct $\left\{G_{B}\left(\mathcal{H}_{K}, C\right), \pi_{\mathcal{H}_{K}}^{C}, \Phi_{\mathcal{H}_{K}}^{C}\right\}$ the GNS triple over $\left\{W\left(\mathcal{H}_{K}\right), C\right\}$ and on it define the operator

$$
B_{Q}(g, t)=\pi_{\mathcal{H}_{K}}^{C} B_{t}(g)
$$

Then $\left\{G_{B}\left(\mathcal{H}_{K}, C\right), \Phi_{\mathcal{H}_{K}}^{C},\left(B_{Q}(g, t)\right)_{t}\right\}$ is a QBM referred to as quantum Brownian motion over $L^{2}(\mathbb{R}, K)$ with covariance $C$, or more loosely with covariance $Q$. We have that

$$
\left\langle\Phi_{\mathcal{H}_{K}}^{C}, B_{Q}(g, t) B_{Q}^{\dagger}(f, s) \Phi_{\mathcal{H}_{K}}^{C}\right\rangle=\varphi_{C}\left(B_{t}(g) B_{s}^{\dagger}(f)\right)=\min (t, s)\left\langle g, \frac{Q+1}{2} f\right\rangle_{K}
$$

and similarly

$$
\left\langle\Phi_{\mathcal{H}_{K}}^{C}, B_{Q}^{\dagger}(f, s) B_{Q}(g, t) \Phi_{\mathcal{H}_{K}}^{C}\right\rangle=\min (t, s)\left\langle g, \frac{Q-1}{2} f\right\rangle_{K} .
$$




\subsection{Quantum Stochastic Calculus}

As is well known, a stochastic calculus can be built up around classical brownian motion and that the resulting theory has widespread applications to studying noisy systems in physics and engineering. It is also possible to build up a quantum stochastic calculus based on the QBMs we have just considered. This was originally done by Hudson and Parthasarathy [13], 14. The basic integrators are $d t$ and, depending on the context, $d B_{t}^{\sharp}$ or $d B_{t}^{\sharp}(g)$ or $d B_{Q}^{\sharp}(t, g)$. In the simplest case, for instance, we have, for a partition $-\infty=t_{1}<t_{2}<\ldots<t_{n}<t_{n+1}=\infty$,

$$
L^{2}(\mathbb{R})=\bigoplus_{m=1}^{n} L^{2}\left(\left[t_{m}, t_{m+1}\right]\right)
$$

and consequently

$$
\Gamma_{B}\left(L^{2}(\mathbb{R})\right)=\bigotimes_{m=1}^{n} \Gamma_{B}\left(L^{2}\left(\left[t_{m}, t_{m+1}\right]\right)\right.
$$

This gives the required time filtration in the quantum situation. We say that a family of operators $\left(X_{t}\right)_{t}$ on $\Gamma_{B}\left(\left(L^{2}(\mathbb{R})\right)\right)$ is adapted if, for all $t$,

$$
X_{t} \equiv \tilde{X}_{t} \otimes 1
$$

on $\Gamma_{B}\left(L^{2}((-\infty, t)) \otimes \Gamma_{B}\left(L^{2}([t, \infty))\right)\right.$. The quantum Ito table reads as

$$
\begin{aligned}
& d B_{Q}(g, t) d B_{Q}^{\dagger}(f, t) \equiv\left\langle g, \frac{Q+1}{2} f\right\rangle_{K} d t, \\
& d B_{Q}^{\dagger}(f, t) d B_{Q}(g, t) \equiv\left\langle g, \frac{Q-1}{2} f\right\rangle_{K} d t, \\
&(d t)^{2}, d t d B_{Q}^{\sharp}(g, t),\left(d B_{Q}^{\sharp}(g, t)\right)^{2} \equiv 0,
\end{aligned}
$$

Let $\left(X_{t}\right)_{t}$ be an adapted process and of the form

$$
X_{t}=\int_{0}^{t}\left(x_{s} d s+x_{s}^{+} d B_{Q}^{\dagger}(g, s)+x_{s}^{-} d B_{Q}(g, s)\right)
$$

and $\left(Y_{t}\right)_{t}$ a similar process, then we have the quantum Ito formula

$$
d\left(X_{t} \cdot Y_{t}\right) \equiv d X_{t} \cdot Y_{t}+X_{t} \cdot d Y_{t}+d X_{t} \cdot d Y_{t}
$$

with

$$
\left.d X_{t}=x_{t} d t+x_{t}^{+} d B_{Q}^{\dagger}(g, t)+x_{t}^{-} d B_{Q}(g, t)\right)
$$

\subsection{The Weak Coupling Limit of Quantum Field Theory}

The results of Accardi, Frigerio and Lu concerning the weak coupling limit for an interaction (31) which has undergone both a dipole and rotating wave approximation can be summarised as follows;

Recall that

$$
\lim _{\lambda \rightarrow 0}\left\langle\Psi_{R}, B_{t / \lambda^{2}}^{(\omega, \lambda)}(g) B_{s / \lambda^{2}}^{(\omega, \lambda) \dagger}(f) \Psi_{R}\right\rangle=\min (t, s) \int_{-\infty}^{\infty} d \tau\left\langle S_{\tau}^{\omega} g, f\right\rangle ;
$$

now define a sesquilinear form $(. \mid .)^{\omega}$ on $\mathcal{H}_{R}^{1}$ the one particle reservoir space by

$$
(g \mid f)^{\omega}=\int_{-\infty}^{\infty} d \tau\left\langle S_{\tau}^{\omega} g, f\right\rangle
$$

We consider the space of suitable test-functions $T^{\omega} \subset \mathcal{H}_{R}^{1}$, determined by the condition

$$
\int_{-\infty}^{\infty} d t\left|\left\langle g, S_{t}^{\omega} f\right\rangle\right|\langle\infty
$$

whenever $f, g \in T^{\omega}$. Note that technically $T^{\omega}$ does not depend on $\omega$ however we keep it in as a label. Then we construct $K_{\omega}$ the completion of $T^{\omega}$ with respect to $(. \mid)^{\omega}$. That is $K^{\omega}$ is the completion of $T^{\omega}$ factored out by its $(. \mid .)^{\omega}$-norm null space. $K^{\omega}$ is a separable Hilbert space with inner product $(. \mid .)^{\omega}$ 
Theorem 1. In the limit $\lambda \rightarrow 0$ the stochastic process on the resevoir space

$$
\left\{\mathcal{H}_{R}, \Psi_{R},\left(B_{t / \lambda^{2}}^{(\omega, \lambda)}(f)\right)_{t}\right\}
$$

for $f \in K^{\omega}$, converges weakly in the sense of matrix elements to $Q B M$ on $L^{2}\left(R, K_{\omega}\right)$. We denote this $Q B M$ by $\left\{H^{\omega}=\right.$ $\left.\Gamma_{B}\left(L^{2}\left(R, K^{\omega}\right)\right), \Phi^{\omega}=\Phi_{K^{\omega}},\left(B_{t}^{\omega}(f)\right)_{t}\right\}$ In the next theorem we show that $U_{t / \lambda^{2}}^{(\lambda)}$ converges to a stochastic process $U_{t}$ on $H_{S} \otimes H^{\omega}$ in a sense to be made explicit now.

Theorem 2. Let $\left.f^{(j)}, h^{\left(j^{\prime}\right)} \in K^{\omega} ; T^{(j)}, S^{\left(j^{\prime}\right)}\right\rangle 0$, for $j=1, \ldots, n: j^{\prime}=1, \ldots, m$ and let $\phi, \phi^{\prime} \in H_{S}$ then the limit as $\lambda \rightarrow 0$ of the matrix element

$$
\left\langle\phi \otimes B_{T^{(1)} / \lambda^{2}}^{(\omega, \lambda) \dagger}\left(f^{(1)}\right) \ldots B_{T^{(m)} / \lambda^{2}}^{(\omega, \lambda) \dagger}\left(f^{(n)}\right) \Psi_{R}\left|U_{t / \lambda^{2}}^{(\lambda)}\right| \phi^{\prime} \otimes B_{S^{(1)} / \lambda^{2}}^{(\omega, \lambda)}\left(h^{(1)}\right) \ldots B_{S^{(m)} / \lambda^{2}}^{(\omega, \lambda) \dagger}\left(h^{(m)}\right) \Psi_{R}\right\rangle
$$

exists and equals

$$
\left\langle\phi \otimes B_{T^{(1)}}^{\omega \dagger}\left(f^{(1)}\right) \ldots B_{T^{(n)}}^{\omega \dagger}\left(f^{(n)}\right) \Phi^{\omega}\left|U_{t}\right| \phi^{\prime} \otimes B_{S^{(1)}}^{\omega \dagger}\left(h^{(1)}\right) \ldots B_{S^{(m)}}^{\omega \dagger}\left(h^{(m)}\right) \Phi^{\omega}\right\rangle,
$$

where $U_{t}$ is a process on $H_{S} \otimes H^{\omega}$ which is the solution to the quantum stochastic differential equation

$$
d U_{t}=\left\{D \otimes d B_{t}^{\omega \dagger}(g)-D^{\dagger} \otimes d B_{t}^{\omega}(g)-(g \mid g)^{\omega-} D^{\dagger} D \otimes d t\right\} U_{t},
$$

with

$$
(g \mid f)^{\omega-}=\int_{-\infty}^{0} d \tau\left\langle g, S_{\tau}^{\omega} f\right\rangle .
$$

Note that $d\left(U_{t} U_{t}^{\dagger}\right) \equiv 0 \equiv d\left(U_{t}^{\dagger} U_{t}\right)$ by the quantum Ito formula and the Ito table. So $U_{t}$ is unitary on $\mathcal{H}_{S} \otimes \mathcal{H}^{\omega}$, however it describes an irreversible evolution when restricted to $\mathcal{H}_{S}$. The unitarity condition corresponds to a fluctuation-dissipation law.

Theorem 3 Let $X \in B\left(H_{S}\right)$, then in the notation of theorem 2 the limit

$$
\begin{gathered}
\left\langle\phi \otimes B_{T^{(1)} / \lambda^{2}}^{(\omega, \lambda) \dagger}\left(f^{(1)}\right) . . B_{T^{(n)} / \lambda^{2}}^{(\omega, \lambda) \dagger}\left(f^{(n)}\right) \Psi_{R}\right| \\
U_{t / \lambda^{2}}^{(\lambda) \dagger}\left(X \otimes 1_{R}\right) U_{t / \lambda^{2}}^{(\lambda)}\left|\phi^{\prime} \otimes B_{S^{(1)} / \lambda^{2}}^{(\omega, \lambda) \dagger}\left(h^{(1)}\right) \ldots B_{S^{(m)} / \lambda^{2}}^{(\omega, \lambda) \dagger}\left(h^{(m)}\right) \Psi_{R}\right\rangle
\end{gathered}
$$

exists and equals

$$
\left\langle\phi \otimes B_{T^{(1)}}^{\omega \dagger}\left(f^{(1)}\right) \ldots B_{T^{(n)}}^{\omega \dagger}\left(f^{(n)}\right) \Phi^{\omega}\left|U_{t}^{\dagger}(X \otimes 1) U_{t}\right| \phi^{\prime} \otimes B_{(1)}^{\omega \dagger}\left(h^{(1)}\right) \ldots B_{(m)}^{\omega \dagger}\left(h^{(m)}\right) \Phi^{\omega}\right\rangle .
$$

Note that in these theorems we encounter vectors of the type $B_{T / \lambda^{2}}^{(\omega, \lambda) \dagger}(f) \Psi_{R}$ which are exponential vectors with test functions $\lambda \int_{0}^{T / \lambda^{2}} d \tau S_{\tau}^{\omega} f$ : they are called collective coherent vectors in the terminology of Accardi and Lu and they are designed to extract the long time cumulative behaviour of the reservoir fields.

\subsection{Non-Zero Temperature Reservoir}

Next, for the non-vacuum case, we consider a density matrix $\rho_{Q}$ on $\mathcal{H}_{\mathcal{R}}$ which is invariant under the free evolution and gaussian with covariance $Q \geq 1_{\mathcal{H}_{R}^{1}}$. That is

$$
\operatorname{Tr}\left\{\rho_{Q} W(g)\right\}=e^{-\frac{1}{2}\langle g, Q g\rangle}, \forall g \in \mathcal{H}_{R}^{1} .
$$

The invariance condition is equivalent to

$$
\left[S_{t}, Q\right]=0, \text { on } \operatorname{Dom}(Q) .
$$

In particular, the choice of a heat bath at inverse temperature $\beta$ and fugacity $z$ is given by

$$
Q=\frac{1+z e^{-\beta H_{R}^{1}}}{1-z e^{-\beta H_{R}^{1}}}
$$

that is

$$
(Q f)(k)=\operatorname{coth} \frac{\beta}{2}(\hbar \omega(k)-\mu) f(k),
$$


where $\mu=\frac{1}{\beta} \ln z$ is the chemical potential.

Now

$$
\begin{gathered}
\lim _{\lambda \rightarrow 0} \operatorname{Tr}\left\{\rho_{Q} B_{t / \lambda^{2}}^{(\omega, \lambda)}(g) B_{s / \lambda^{2}}^{(\omega, \lambda)}(f)\right\}=\min (t, s) \int_{-\infty}^{\infty} d \tau \operatorname{Tr}\left\{\rho_{Q} A\left(S_{\tau}^{\omega} g\right) A^{\dagger}(f)\right\} \\
=\min (t, s) \int_{-\infty}^{\infty} d \tau\left\langle S_{\tau}^{\omega} g,\left(\frac{Q+1}{2}\right) f\right\rangle
\end{gathered}
$$

and similarly

$$
\lim _{\lambda \rightarrow 0} \operatorname{Tr}\left\{\rho_{Q} B_{s / \lambda^{2}}^{(\omega \lambda) \dagger}(f) B_{t / \lambda^{2}}^{(\omega, \lambda)}(g)\right\}=\min (t, s) \int_{-\infty}^{\infty} d \tau\left\langle S_{\tau}^{\omega} g,\left(\frac{Q-1}{2}\right) f\right\rangle .
$$

Let $T_{Q}^{\omega}$ be the subset of $\operatorname{Dom}(Q)$ such that

$$
\int_{-\infty}^{\infty}\left|\left\langle f, S_{t}^{\omega} h\right\rangle\right| d t<\infty \text { and } \int_{-\infty}^{\infty}\left|\left\langle f, S_{t}^{\omega} Q h\right\rangle\right| d t<\infty
$$

whenever $f, h \in T_{Q}^{\omega}$. Let $K_{Q}^{\omega}$ be the Hilbert space completion of $T_{Q}^{\omega}$ with respect to the sesquilinear form (.|.) $)_{Q}^{\omega}$ given by

$$
(f \mid h)_{Q}^{\omega}=\int_{-\infty}^{\infty}\left\langle f, Q S_{t}^{\omega} h\right\rangle d t
$$

Note that in most cases $T_{Q}^{\omega}$ is dense in $\mathcal{H}_{R}^{1}$ and that $K_{Q}^{\omega}$ is a Hilbert space equipped with inner product (.|. $)_{Q}^{\omega}$.

Theorem 1a: The process $\left(B_{t / \lambda^{2}}^{(\lambda)}(f)\right)_{t}$ in the mixed state $\rho_{Q}$ converges weakly in the sense of matrix elements to $Q B M$ over $L^{2}\left(R, K_{Q}^{\omega}\right)$ with covariance $Q$. This is denoted as $\left\{H_{Q}^{\omega}=G_{B}\left(\left(L^{2}\left(R, K_{Q}^{\omega}\right), Q \otimes 1\right), \Phi_{Q}^{\omega},\left(B_{Q}^{\omega}(f, t)\right)_{t}\right\}\right.$.

Theorem 2a; For $\phi, \phi^{\prime} \in H_{S}, f^{(j)}, h^{\left(j^{\prime}\right)} \in K_{Q}^{\omega}$ and $\left.T^{(j)}, S^{\left(j^{\prime}\right)}\right\rangle 0$, for $j=1, \ldots n ; j^{\prime}=1, \ldots, m$, the limit as $\lambda \rightarrow 0$ of

$$
\operatorname{Tr}\left\{\left|\phi^{\prime}\right\rangle\langle\phi| \otimes B_{S^{(m)} / \lambda^{2}}^{(\omega, \lambda) \dagger}\left(h^{(m)}\right) \ldots B_{S^{(1)} / \lambda^{2}}^{(\omega, \lambda \dagger}\left(h^{(1)}\right) \rho_{Q} B_{T^{(1)} / \lambda^{2}}^{(\omega, \lambda)}\left(f^{(1)}\right) \ldots B_{T^{(n)} / \lambda^{2}}^{(\omega, \lambda)}\left(f^{(n)}\right) U_{t / \lambda^{2}}^{(\lambda)}\right\}
$$

exists and equals

$$
\left\langle\phi \otimes B_{Q}^{\omega \dagger}\left(f^{(1)}, T^{(1)}\right) \ldots B_{Q}^{\omega \dagger}\left(f^{(n)}, T^{(n)}\right) \Phi_{Q}^{\omega}\left|U_{t}\right| \phi^{\prime} \otimes B_{Q}^{\omega \dagger}\left(h^{(1)}, S^{(1)}\right) \ldots B_{Q}^{\omega \dagger}\left(h^{(m)}, S^{(m)}\right) \Phi_{Q}^{\omega}\right\rangle
$$

where $U_{t}$ is a unitary operator on $H_{S} \otimes G_{B}\left(L^{2}\left(R, K_{Q}^{\omega}\right), C\right)$, with $C=Q \otimes 1$, satisfying the QSDE

$$
d U_{t}=\left\{D \otimes d B_{Q}^{\omega \dagger}(g, t)-D^{\dagger} \otimes d B_{Q}^{\omega}(g, t)-(g \mid g)_{Q+}^{\omega-} D^{\dagger} D \otimes d t-\overline{(g \mid g)}_{Q-}^{\omega-} D D^{\dagger} \otimes d t\right\} U_{t},
$$

where

$$
(g \mid f)_{Q \pm}^{\omega-}=\int_{-\infty}^{0} d \tau\left\langle g, S_{\tau}^{\omega} \frac{Q \pm 1}{2} f\right\rangle .
$$

Theorem 3a: For $X \in B\left(H_{S}\right)$, then in the notation of theorem 2

$$
\lim _{\lambda \rightarrow 0} \operatorname{Tr}\left\{\left|\phi^{\prime}\right\rangle\langle\phi| \otimes B_{S^{(m)} / \lambda^{2}}^{(\omega, \lambda) \dagger}\left(h^{(m)}\right) \ldots B_{S^{(1)} / \lambda^{2}}^{(\omega, \lambda) \dagger}\left(h^{(1)}\right) \rho_{Q} B_{T^{(1)} / \lambda^{2}}^{(\omega, \lambda)}\left(f^{(1)}\right) \ldots B_{T^{(n)} / \lambda^{2}}^{(\omega, \lambda)}\left(f^{(n)}\right) u_{t / \lambda^{2}}^{(\lambda)}(X \otimes 1)\right\}
$$

exists and equals

$$
\left\langle\phi \otimes B_{Q}^{\omega \dagger}\left(f^{(1)}, T^{(1)}\right) \ldots B_{Q}^{\omega \dagger}\left(f^{(n)}, T^{(n)}\right) \Phi_{Q}^{\omega}\left|U_{t}^{\dagger}(X \otimes 1) U_{t}\right| \phi^{\prime} \otimes B_{Q}^{\omega \dagger}\left(h^{(1)}, S^{(1)}\right) \ldots B_{Q}^{\omega \dagger}\left(h^{(m)}, S^{(m)}\right) \Phi_{Q}^{\omega}\right\rangle .
$$




\subsection{The Quantum Stochastic Limit for the Full Interaction}

Now suppose that the interaction is of the form (31). The problem of dropping the rotating wave approximation was first tackled by Accardi and $\mathrm{Lu}$ in [10] for an interaction similar to (31) except that all the test functions were taken to be the same. The result is that for each Bohr frequency $\omega$ we obtain a separate independent QBM.

First of all note that $\Gamma_{B}\left(\bigoplus_{\omega \in F} L^{2}\left(\mathbb{R}, K_{Q}^{\omega}\right)\right)=\bigotimes_{\omega \in F} \Gamma_{B}\left(L^{2}\left(\mathbb{R}, K_{Q}^{\omega}\right)\right)$ then consider the Weyl algebra $W\left(\bigoplus_{\omega \in F} L^{2}\left(\mathbb{R}, K_{Q}^{\omega}\right)\right)=$ $\bigotimes_{\omega \in F} W\left(L^{2}\left(\mathbb{R}, K_{Q}^{\omega}\right)\right)$, with quasi-free state $\varphi_{\tilde{C}}$ with covariance $\tilde{C}=\bigotimes_{\omega \in F} C$, where $C=Q \otimes 1$ on $\mathcal{H}_{K_{Q}^{\omega}} \cong K_{Q}^{\omega} \otimes L^{2}(\mathbb{R})$. The GNS triple over $\left\{W\left(\bigoplus_{\omega \in F} L^{2}\left(\mathbb{R}, K_{Q}^{\omega}\right)\right), \varphi_{\tilde{C}}\right\}$ is $\left.\left\{\mathcal{H}_{Q}^{\omega}=\Gamma_{B}\left(\bigoplus_{\omega \in F} L^{2}\left(\mathbb{R}, K_{Q}^{\omega}\right)\right), \tilde{C}\right)\right), \pi_{Q}^{F}$, $\left.\Phi_{Q}^{\omega}\right\}$. Now observe that

$$
\mathcal{H}_{Q}^{F}=\bigotimes_{\omega \in F} \mathcal{H}_{Q}^{\omega}, \pi_{Q}^{F}=\bigotimes_{\omega \in F} \pi_{Q}^{\omega} ; \Phi_{Q}^{F}=\bigotimes_{\omega \in F} \Phi_{Q}^{\omega}
$$

For each $f \in K_{Q}^{\omega}$ we have

$$
B_{Q}^{\omega}(f, t)=\pi_{Q}^{\omega} A_{Q}^{\omega}\left(f \otimes \chi_{[0, t]}\right)
$$

where $A_{Q}^{\omega}$ is annihilation operator on $L^{2}\left(\mathbb{R}, K_{Q}^{\omega}\right)$, so for $\left.t_{\omega}\right\rangle 0, f_{\omega} \in K_{Q}^{\omega}$, for each $\omega \in F$, we have

$$
B_{Q}^{F}\left(\bigotimes_{\omega \in F} f_{\omega},\left(t_{\omega}\right)_{\omega \in F}\right)=\bigotimes_{\omega \in F} B_{Q}^{\omega}\left(f_{\omega}, t_{\omega}\right)
$$

Theorem 1b: For each $\omega \in F$ and $f \in K_{Q}^{\omega}$ the limit $\lambda \rightarrow 0, B_{t / \lambda^{2}}^{(\omega, \lambda)}(f)$ taken in the state $\rho_{Q}$ converges in the sense of matrix elements to $Q B M$ over $L^{2}\left(R, K_{Q}^{\omega}\right)$ with covariance $Q$ and each of these limiting processes are independent for different values of $\omega$.

Theorem 2b: Let $\left.f_{\omega}^{(j)}, h_{\omega}^{\left(j^{\prime}\right)} \in K_{Q}^{\omega} ; T_{\omega}^{(j)}, S_{\omega}^{\left(j^{\prime}\right)}\right\rangle 0$ for each $\omega \in F j=1, \ldots, n_{\omega} ; j^{\prime}=1, \ldots, m_{\omega} ;$ and $t \geq 0 ; \phi, \phi^{\prime} \in H_{S}$ then the limit as $\lambda \rightarrow 0$ of the matrix element

$$
\operatorname{Tr}\left\{\left|\phi^{\prime}\right\rangle\langle\phi| \otimes\left[\bigotimes_{\omega \in F} B_{S_{\omega}^{\left(m_{\omega}\right) / \lambda^{2}}}^{(\omega, \lambda)}\left(h_{\omega}^{\left(m_{\omega}\right)}\right) \ldots B_{S_{\omega}^{(1)} / \lambda^{2}}^{(\omega, \lambda)}\left(h_{\omega}^{(1)}\right)\right]^{\dagger} \times \rho_{Q}\left[\bigotimes_{\omega \in F} B_{T_{\omega}^{(1)} / \lambda^{2}}^{(1, \lambda)}\left(f_{\omega}^{(1)}\right) \ldots B_{T_{\omega}^{\left(n_{\omega}\right)} / \lambda^{2}}^{(\omega, \lambda)}\left(f_{\omega}^{\left(n_{\omega}\right)}\right)\right] U_{t / \lambda^{2}}^{(\lambda)}\right\}
$$

exists and equals

$$
\begin{gathered}
\left\langle\phi \otimes\left[\bigotimes_{\omega \in F} B_{Q}^{\omega}\left(f_{\omega}^{(1)}, T_{\omega}^{(1)}\right) \ldots B_{Q}^{\omega}\left(f_{\omega}^{\left(n_{\omega}\right)}, T_{\omega}^{\left(n_{\omega}\right)}\right)\right]^{\dagger} \Phi_{Q}^{F}\right| \\
U_{t}\left|\phi^{\prime} \otimes\left[\bigotimes_{\omega \in F} B_{Q}^{\omega}\left(h_{\omega}^{(1)}, S_{\omega}^{(1)}\right) \ldots B_{Q}^{\omega}\left(h_{\omega}^{\left(m_{\omega}\right)}, S_{\omega}^{\left(m_{\omega}\right)}\right)\right]^{\dagger} \Phi_{Q}^{F}\right\rangle,
\end{gathered}
$$

where $U_{t}$ is unitary on $H_{S} \otimes H_{Q}^{F}$ and satisfies quantum stochastic differential equation

$$
\begin{gathered}
d U_{t}=\left\{\sum_{\omega \in F} \sum_{j=1}^{N(\omega)}\left[D_{j}^{\omega} \otimes d B_{\omega}^{\dagger}\left(g_{j}^{\omega}, t\right)-D_{j}^{\omega \dagger} \otimes d B_{\omega}\left(g_{j}^{\omega}, t\right)\right]\right. \\
-\sum_{\omega \in F} \sum_{j, k=1}^{N(\omega)} D_{j}^{\omega \dagger} D_{k}^{\omega}\left(g_{j}^{\omega} \mid g_{k}^{\omega}\right)_{Q+}^{\omega-} d t-\sum_{\omega \in F} \sum_{j, k=1}^{N(\omega)} D_{j}^{\omega} D_{k}^{\omega \dagger}{\left.\overline{\left(g_{j}^{\omega} \mid g_{k}^{\omega}\right)_{Q-}^{\omega-}} d t\right\} U_{t},}^{\omega-}
\end{gathered}
$$

with $U_{0}=1$. The coefficients are given by

$$
(f \mid h)_{Q \pm}^{\omega-}=\int_{-\infty}^{0} d t\left\langle f, S_{t}^{\omega}\left(\frac{Q \pm 1}{2}\right) h\right\rangle
$$

for $f, h \in K_{\omega}$. The Ito table is given by

$$
\begin{aligned}
& d B_{Q}^{\omega}(f, t) d B_{Q}^{\omega^{\prime} \dagger}(g, t) \equiv \delta_{\omega, \omega^{\prime}}(f \mid g)_{Q+}^{\omega} d t \\
& d B_{Q}^{\omega \dagger}(g, t) d B_{Q}^{\omega^{\prime}}(f, t) \equiv \delta_{\omega, \omega^{\prime}}(f \mid g)_{Q_{-}}^{\omega} d t .
\end{aligned}
$$

The coefficients in (2.5:8) are

$$
(f \mid g)_{Q+}^{\omega}=\int_{-\infty}^{\infty} d t\left\langle f, S_{t}^{\omega}\left(\frac{Q+1}{2}\right) g\right\rangle=(f \mid g)_{Q+}^{\omega-}+\overline{(g \mid f)_{Q+}^{\omega-}}
$$




$$
(f \mid g)_{Q-}^{\omega}=\int_{-\infty}^{\infty} d t\left\langle f, S_{t}^{\omega}\left(\frac{Q-1}{2}\right) g\right\rangle=\overline{(g \mid f)_{Q-}^{\omega-}}+(f \mid g)_{Q-}^{\omega-} .
$$

The proof of theorem 2a is as follows; first of all we know that for different $\omega$ we can always consider independent Q-quantum Brownian motions. This is done in [10. The next step is to consider the effect of the degeneracy which may arise for each $\omega \in F$. In this case we must, therefore, generalize the results of [8], [10] accordingly. This is done in appendices B and C.

Theorem 3a. In the notations of theorem 2a, for any $X \in B\left(H_{S}\right)$, the limit as $\lambda \rightarrow 0$

$$
\operatorname{Tr}\left\{\left|\phi^{\prime}\right\rangle\langle\phi| \otimes\left[\bigotimes_{\omega \in F} B_{S_{\omega}^{(1)} / \lambda^{2}}^{(\omega, \lambda)}\left(h_{\omega}^{(1)}\right) \ldots B_{S_{\omega}^{(m, \lambda)} / \lambda^{2}}^{\left(m_{\omega}\right)}\left(h_{\omega}^{\left(m_{\omega}\right)}\right)\right]^{\dagger} \rho_{Q}\left[\bigotimes_{\omega \in F} B_{T_{\omega}^{(1)} / \lambda^{2}}^{(\omega, \lambda)}\left(f_{\omega}^{(1)}\right) \ldots B_{T_{\omega}^{(n \omega)} / \lambda^{2}}^{(\omega, \lambda)}\left(f_{\omega}^{\left(n_{\omega}\right)}\right)\right] u_{t / \lambda^{2}}^{(\lambda)}(X \otimes 1)\right\}
$$

exists and equals

$$
\left\langle\phi \otimes\left[\bigotimes_{\omega \in F} B_{Q}^{\omega}\left(f_{\omega}^{(1)}, T_{\omega}^{(1)}\right) \ldots B_{Q}^{\omega}\left(f_{\omega}^{\left(n_{\omega}\right)}, T_{\omega}^{\left(n_{\omega}\right)}\right)\right]^{\dagger} \Phi_{Q}^{F}\left|U_{t}^{\dagger}(X \otimes 1) U_{t}\right| \phi^{\prime} \otimes\left[\bigotimes_{\omega \in F} B_{Q}^{\omega}\left(h_{\omega}^{(1)}, S_{\omega}^{(1)}\right) \ldots B_{Q}^{\omega}\left(h_{\omega}^{\left(m_{\omega}\right)}, S_{\omega}^{\left(m_{\omega}\right)}\right)\right]^{\dagger} \Phi_{Q}^{F}\right\rangle,
$$

where $U_{t}$ is the solution to the quantum stochastic differential equation of theorem.

\subsection{The Langevin and Master Equations}

In each of the cases the right hand side of the expression for $d U_{t}$ contains a term of the form $-Y \otimes d t U_{t}$. For instance, we have

$$
Y=\sum_{\omega \in F} \sum_{j, k=1}^{N(\omega)}\left\{D_{j}^{\omega \dagger} D_{k}^{\omega}\left(g_{j}^{\omega} \mid g_{k}^{\omega}\right)_{Q+}^{\omega-}+D_{j}^{\omega} D_{k}^{\omega \dagger}{\overline{\left(g_{j}^{\omega} \mid g_{k}^{\omega}\right)_{Q-}^{\omega-}}}^{\omega-}\right\},
$$

The Langevin equation then reads as follows

$$
\begin{aligned}
d\left[U_{t}^{\dagger}(X \otimes 1) U_{t}\right] & \equiv\left[d U_{t}\right]^{\dagger}(X \otimes 1) U_{t}+U_{t}^{\dagger}(X \otimes 1) d U_{t}+\left[d U_{t}\right]^{\dagger}(X \otimes 1) d U_{t} \\
\equiv & U_{t}^{\dagger}\left[L_{0}(X) \otimes d t+\sum_{\omega \in F} \sum_{j, k=1}^{N(\omega)} L_{j+}^{\omega}(X) \otimes d B_{Q}^{\omega \dagger}\left(g_{j}^{\omega}, t\right)\right. \\
& \left.+\sum_{\omega \in F} \sum_{j, k=1}^{N(\omega)} L_{j-}^{\omega}(X) \otimes d B_{Q}^{\omega}\left(g_{j}^{\omega}, t\right)\right\} U_{t},
\end{aligned}
$$

where

$$
L_{0}(X)=-X Y-Y^{\dagger} X+\Theta(X)
$$

with

$$
\begin{gathered}
\Theta(X)=\sum_{\omega \in F} \sum_{j, k=1}^{N(\omega)}\left\{D_{j}^{\omega \dagger} X D_{k}^{\omega}\left[\left(g_{j}^{\omega} \mid g_{k}^{\omega}\right)_{Q+}^{\omega-}+\overline{\left(g_{k}^{\omega} \mid g_{j}^{\omega}\right)_{Q+}^{\omega-}}\right]+D_{j}^{\omega} X D_{k}^{\omega \dagger}\left[\overline{\left(g_{j}^{\omega} \mid g_{k}^{\omega}\right)_{Q-}^{\omega-}}+\left(g_{k}^{\omega} \mid g_{j}^{\omega}\right)_{Q-}^{\omega-}\right]\right\} \\
\left.=\sum_{\omega \in F} \sum_{j, k=1}^{N(\omega)}\left\{D_{j}^{\omega \dagger} X D_{k}^{\omega}\left(g_{j}^{\omega} \mid g_{k}^{\omega}\right)_{Q+}^{\omega}+D_{k}^{\omega} X D_{j}^{\omega \dagger}\left(g_{j}^{\omega} \mid g_{k}^{\omega}\right)_{Q-}^{\omega}\right]\right\}
\end{gathered}
$$

and

$$
L_{j+}^{\omega}(X)=X D_{j}^{\omega}-D_{j}^{\omega} X, L_{j-}^{\omega}(X)=D_{j}^{\omega \dagger} X-X D_{j}^{\omega \dagger} .
$$

Note that unitarity follows from

$$
L_{0}\left(1_{S}\right)=-\left(Y+Y^{\dagger}\right)+\Theta\left(1_{S}\right)=0 ; L_{j \pm}^{\omega}\left(1_{S}\right)=0 .
$$

It is instructive to set $Y=\frac{1}{2} \Gamma+\frac{i}{\hbar} H_{S}^{\prime}$ where both $\Gamma$ and $H_{S}^{\prime}$ are self-adjoint; we then have that

$$
L_{0}(X)=-\frac{1}{2}(X \Gamma+\Gamma X)+\Theta(X)+\frac{1}{i \hbar}\left[X, H_{S}^{\prime}\right]
$$


The unitarity condition is then $2 \operatorname{Re} Y=\Gamma=\Theta\left(1_{S}\right)$; this is the fluctuation-dissipation relation. The presence of the imaginary term $H_{S}^{\prime}$ does not effect the unitarity. For $\rho_{S}$ a density matrix on $\mathcal{H}_{S}$ we define the expectation $\langle.\rangle_{t}$ by

$$
\langle X\rangle_{t}=\operatorname{Tr}\left\{\rho_{S} \otimes\left|\Phi_{Q}^{F}\right\rangle\left\langle\Phi_{Q}^{F}\right| U_{t}^{\dagger}(X \otimes 1) U_{t}\right\}=\operatorname{Tr}\left\{s_{t} X\right\},
$$

where $s_{t}$ denotes the effective density matrix on $(S)$ and the second trace is a partial trace over the system space (the trace over the reservoir space assumed to be taken already). Now

$$
\frac{d}{d t}\langle X\rangle_{t}=\operatorname{Tr}\left\{\rho_{S} \otimes\left|\Phi_{Q}^{F}\right\rangle\left\langle\Phi_{Q}^{F}\right| \frac{d}{d t} U_{t}^{\dagger}(X \otimes 1) U_{t}\right\}
$$

so in terms of the effective density matrix $s_{t}$

$$
\frac{d}{d t} \operatorname{Tr}\left\{s_{t} X\right\}=\operatorname{Tr}\left\{s_{t} L_{0}(X)\right\}=\operatorname{Tr}\left\{L_{0}^{*}\left(s_{t}\right) X\right\},
$$

where $L_{0}^{*}$ denotes the adjoint operation to $L_{0}$ on the dual of $\mathcal{B}\left(\mathcal{H}_{S}\right)$. This gives the master equation

$$
\begin{gathered}
\frac{d s_{t}}{d t}=L_{0}^{*}\left(s_{t}\right)=-\sum_{\omega \in F} \sum_{j, k=1}^{N(\omega)}\left\{\left[D_{j}^{\omega} D_{k}^{\omega \dagger} s_{t}-D_{k}^{\omega \dagger} s_{t} D_{j}^{\omega}\right] \overline{\left(g_{j}^{\omega} \mid g_{k}^{\omega}\right)_{Q-}^{\omega-}}+\left[D_{j}^{\omega \dagger} D_{k}^{\omega} s_{t}-D_{k}^{\omega} s_{t} D_{j}^{\omega \dagger}\right]\left(g_{j}^{\omega} \mid g_{k}^{\omega}\right)_{Q+}^{\omega-}\right. \\
\left.-\left[D_{j}^{\omega \dagger} s_{t} D_{k}^{\omega-}-s_{t} D_{k}^{\omega} D_{j}^{\omega \dagger}\right]\left(g_{j}^{\omega} \mid g_{k}^{\omega}\right)_{Q-}^{\omega-}-\left[D_{j}^{\omega} s_{t} D_{k}^{\omega \dagger}-s_{t} D_{k}^{\omega \dagger} D_{j}^{\omega}\right] \overline{\left(g_{j}^{\omega} \mid g_{k}^{\omega}\right)_{Q+}^{\omega-}}\right\} .
\end{gathered}
$$

From the relation (31) we see that $\left[Y, H_{S}\right]=0$. If we define the effective evolution operator by

$$
V_{t}=\left(e^{\frac{t}{i \hbar} H_{S}} \otimes 1\right) U_{t}
$$

which satisfies the quantum stochastic differential equation

$$
d V_{t} \equiv\left(\frac{1}{i \hbar} H_{S} e^{\frac{t}{i \hbar} H_{S}} \otimes d t\right) U_{t}+\left(e^{\frac{t}{i \hbar} H_{S}} \otimes 1\right) d U_{t}
$$

Explicitly this gives

$$
\begin{aligned}
d V_{t} & \equiv\left(e^{\frac{t}{2 \hbar} H_{S}} \otimes 1\right)\left[\sum_{\omega \in F} \sum_{\phi, \phi^{\prime} \in B}^{\left(\omega_{\phi \phi^{\prime}}=\omega\right)}\left\{T_{\phi \phi^{\prime}} \otimes d B_{Q}^{\omega \dagger}\left(g_{\phi \phi^{\prime}}, t\right)-T_{\phi \phi^{\prime}}^{\dagger} \otimes d B_{Q}^{\omega}\left(g_{\phi \phi^{\prime}}, t\right)\right\}-\left\{Y+\frac{i}{\hbar} H_{S}\right\} d t\right] U_{t} 1 \\
& \equiv\left[\sum_{\omega \in F} \sum_{\phi, \phi^{\prime} \in B}^{\left(\omega_{\phi \phi^{\prime}}=\omega\right)}\left\{T_{\phi \phi^{\prime}} \otimes d B_{Q}^{\omega \dagger}\left(g_{\phi \phi^{\prime}}, t\right) e^{i \omega t}-T_{\phi \phi^{\prime}}^{\dagger} \otimes d B_{Q}^{\omega}\left(g_{\phi \phi^{\prime}}, t\right) e^{-i \omega t}\right\}-\left\{\frac{1}{2} \Gamma+\frac{i}{\hbar}\left(H_{S}+H^{\prime}{ }_{S}\right)\right\} d t\right] V_{t} .
\end{aligned}
$$

We see that $H^{\prime}{ }_{S}$ is a physical addition to the system Hamiltonian due to the presence of the quantum field. This is precisely the Lamb shift. Since $Y$ commutes with $H_{S}$ it is enough to compute its expectations for eigenstates $\phi \in B$;

$$
\langle\phi, Y \phi\rangle=\sum_{\omega \in F} \sum_{j, k=1}^{N(\omega)}\left\{\left\langle\phi, D_{j}^{\omega \dagger} D_{k}^{\omega} \phi\right\rangle\left(g_{j}^{\omega} \mid g_{k}^{\omega}\right)_{Q+}^{\omega-}+\left\langle\phi, D_{j}^{\omega} D_{k}^{\omega \dagger} \phi\right\rangle{\overline{\left(g_{j}^{\omega} \mid g_{k}^{\omega}\right)_{Q-}}}_{Q-}^{\omega}\right\} .
$$

This is however equivalent to the (complex) shift one calculates using second order perturbation theory. For example, taking the zero temperature for simplicity, one calculates in second order shift [19]

$$
\begin{aligned}
Y_{\phi}^{(2)} & =\frac{1}{i \hbar}\left\langle\phi \otimes \Psi_{R}, H_{I} \frac{1}{H^{(0)}-E_{\phi}-i 0^{+}} H_{I} \phi \otimes \Psi_{R}\right\rangle \\
& =\sum_{\omega, \omega^{\prime} \in F} \sum_{j}^{N(\omega)} \sum_{j^{\prime}}^{N\left(\omega^{\prime}\right)} \int_{-\infty}^{0} d \tau\left\langle\phi \otimes \Psi_{R}, D_{j}^{\omega} \otimes A\left(g_{j}^{\omega}\right) e^{i\left(H^{(0)}-E_{\phi}\right) \tau / \hbar} D_{j^{\prime}}^{\omega^{\prime}} \otimes A^{\dagger}\left(g_{j^{\prime}}^{\omega^{\prime}}\right) \phi \otimes \Psi_{R}\right\rangle .
\end{aligned}
$$

Here we have used the well known identity

$$
\int_{-\infty}^{0} d t e^{i x t}=\frac{1}{i\left(x-i 0^{+}\right)}=\pi \delta(x)-i \wp\left(\frac{1}{x}\right) ;(x \in \mathbb{R}),
$$


where $\wp$ means that we take the principal part of the integral. Now $D_{j}^{\omega} \phi$ is an eigenstate of $H_{S}$ with eigenvalue $E_{\phi}-\hbar \omega$, so the summation need only be considered over $\omega=\omega^{\prime}$ in $(2.6: 17)$ above. Therefore we have

$$
\begin{aligned}
Y_{\phi}^{(2)} & =\sum_{\omega \in F} \sum_{j, k}^{N(\omega)} \int_{-\infty}^{0} d \tau\left\langle\phi \otimes \Psi_{R}, D_{j}^{\omega \dagger} \otimes A\left(g_{j}^{\omega}\right) e^{i\left(H_{R}-\hbar \omega\right) \tau / \hbar} D_{k}^{\omega} \otimes A^{\dagger}\left(g_{k}^{\omega}\right) \phi \otimes \Psi_{R}\right\rangle \\
& =\sum_{\omega \in F} \sum_{j, k}^{N(\omega)}\left\langle\phi, D_{j}^{\omega \dagger} D_{k}^{\omega} \phi\right\rangle \int_{-\infty}^{0} d \tau\left\langle\Psi_{R}, A\left(g_{j}^{\omega}\right) A^{\dagger}\left(S_{\tau}^{\omega} g_{k}^{\omega}\right) \Psi_{R}\right\rangle .
\end{aligned}
$$

Hence $Y_{\phi}^{(2)}=\langle\phi, Y \phi\rangle$. The real and imaginary parts of $Y$ are therefore the linewidth and energy shift as would normally be calculated using second order perturbation theory, this is true in the non-vacuum cases also.

\subsection{Transition Probabilities}

Let $P_{t}(\psi \mid \phi)$ denote the probability that the sytem will be measured in state $\psi$ at time $t$ if it initially was in state $\phi$, then

$$
p_{t}(\psi \mid \phi)=\left\langle\phi \otimes \Phi_{Q}^{F}, U_{t}^{\dagger}(|\psi\rangle\langle\psi| \otimes 1) U_{t} \phi \otimes \Phi_{Q}^{F}\right\rangle
$$

from theorem 3,3a or $3 \mathrm{~b}$ we have

$$
\frac{d}{d t} p_{t}(\psi \mid \phi)=\left\langle\phi \otimes \Phi_{Q}^{F}, U_{t}^{\dagger} L_{0}(|\phi\rangle\langle\phi|) \otimes 1 U_{t} \phi \otimes \Phi_{Q}^{F}\right\rangle .
$$

Therefore, setting $t=0$,

$$
\left.\frac{d}{d t} p_{t}(\psi \mid \phi)\right|_{t=0}=\left\langle\phi, L_{0}(|\psi\rangle\langle\psi|) \phi\right\rangle
$$

If $\psi=\phi$ we obtain the relation

$$
\left.\frac{d}{d t} p_{t}(\phi \mid \phi)\right|_{t=0}=-\langle\phi, \Gamma \phi\rangle
$$

while if $\langle\phi, \psi\rangle=0$ then (112) gives

$$
\begin{gathered}
\left.\frac{d}{d t} p_{t}(\psi \mid \phi)\right|_{t=0}=\langle\phi, \Theta(|\psi\rangle\langle\psi|) \phi\rangle \\
=\sum_{\omega \in F} \sum_{j, k=1}^{N(\omega)}\left\{\left\langle\phi, D_{j}^{\omega \dagger} \psi\right\rangle\left\langle\psi, D_{k}^{\omega} \phi\right\rangle\left(g_{j}^{\omega} \mid g_{k}^{\omega}\right)_{Q+}^{\omega}+\left\langle\phi, D_{k}^{\omega} \psi\right\rangle\left\langle\psi, D_{j}^{\omega \dagger} \phi\right\rangle\left(g_{j}^{\omega} \mid g_{k}^{\omega}\right)_{Q-}^{\omega}\right\},
\end{gathered}
$$

Using the relation $\int_{-\infty}^{\infty} d t e^{i x t}=2 \pi \delta(x)$ we can rewrite this as

$$
\begin{gathered}
\left.\frac{d}{d t} p_{t}(\psi \mid \phi)\right|_{t=0}=2 \pi \sum_{\omega \in F} \sum_{j, k=1}^{N(\omega)} \int d k \overline{g_{j}^{\omega}}(\mathbf{k}) g_{k}^{\omega}(\mathbf{k}) \delta(\omega(k)-\omega) \\
\left\{\left\langle\phi, D_{j}^{\omega \dagger} \psi\right\rangle\left\langle\psi, D_{k}^{\omega} \phi\right\rangle \frac{q(\mathbf{k})+1}{2}+\left\langle\phi, D_{k}^{\omega} \psi\right\rangle\left\langle\psi, D_{j}^{\omega \dagger} \phi\right\rangle \frac{q(\mathbf{k})-1}{2}\right\},
\end{gathered}
$$

where $q(\mathbf{k})$ is the spectral function associated with $Q$, cf (125). This is our formulation of the Fermi golden rule for transitions of the systems state and it corresponds to the usual expressions, cf formulae (1.21.27a,b) of [2].

\section{THE WEAK COUPLING LIMIT IN QED.}

As an illustration of our theory we consider the case of quantum electrodynamics. We stress however that the theory encompasses a wide range of physical phenomena. For instance exciton models in solid state physics such as phonon models or the Frölich [15, 16] polaron model will differ from the following treatment in only minor technical details. The electromagnetic field acts as reservoir for our system $(S)$ which we take to consist of a single electron. The electromagnetic field can be derived from the potential $\mathbf{A}$ given by

$$
\mathbf{A}(\mathbf{r})=\sum_{\sigma=1,2} \int \frac{d^{3} k}{(2 \pi)^{3 / 2}} \sqrt{\frac{\hbar}{2 \epsilon_{o} c|\mathbf{k}|}}\left\{a_{\sigma}^{\dagger}(\mathbf{k}) e^{-i \mathbf{k} \cdot \mathbf{r}}+a_{\sigma}(\mathbf{k}) e^{i \mathbf{k} \cdot \mathbf{r}}\right\} \boldsymbol{\epsilon}^{\sigma}(\hat{\mathbf{k}}) .
$$


Here we consider two transverse polarizations $(\sigma=1,2)$ for each mode $\mathbf{k}$. In our notation $\left\{\boldsymbol{\epsilon}^{1}(\hat{\mathbf{k}}), \boldsymbol{\epsilon}^{2}(\hat{\mathbf{k}}), \hat{\mathbf{k}}=|\mathbf{k}|^{-1} \mathbf{k}\right\}$ form a right-handed triad for each $\mathbf{k}$. This ensures that we are working with the radiation gauge $\nabla \cdot \mathbf{A}=0$. The operators $a_{\sigma}^{\sharp}(\mathbf{k})$ on the reservoir state space $\mathcal{H}_{R}$ satisfy Bose commutation relations

$$
\left[a_{\sigma}(\mathbf{k}), a_{\sigma^{\prime}}^{\dagger}\left(\mathbf{k}^{\prime}\right)\right]=\delta_{\sigma \sigma^{\prime}} \delta\left(\mathbf{k}-\mathbf{k}^{\prime}\right)
$$

The total Hamiltonian for the system and reservoir is

$$
\begin{aligned}
H= & \frac{1}{2 m}|\mathbf{p}-e \mathbf{A}|^{2}+\Phi(\mathbf{r})+H_{R} \\
& =H_{S}+H_{R}+H_{I}+H^{\prime}{ }_{I},
\end{aligned}
$$

where the unperturbed system Hamiltonian (with potential $\Phi(\mathbf{r}))$ is

$$
\begin{gathered}
H_{S}=\frac{1}{2 m}|\mathbf{p}|^{2}+\Phi(\mathbf{r}) ; \\
H_{R}=\sum_{\sigma=1,2} \int d^{3} k \hbar c|k| a_{\sigma}^{\dagger}(\mathbf{k}) a_{\sigma}(\mathbf{k}), \\
H_{I}=-\frac{e}{m} \sum_{\sigma=1,2} \int d^{3} k\left\{a_{\sigma}^{\dagger}(\mathbf{k}) e^{-i \mathbf{k} \cdot \mathbf{r}}+a_{\sigma}(\mathbf{k}) e^{i \mathbf{k} \cdot \mathbf{r}}\right\} \mathbf{G}^{\sigma}(\mathbf{k}) \cdot \mathbf{p},
\end{gathered}
$$

with

$$
\mathbf{G}^{\sigma}(\hat{\mathbf{k}})=\frac{1}{(2 \pi)^{3 / 2}} \sqrt{\frac{\hbar}{2 \epsilon_{o} c|\mathbf{k}|}} \boldsymbol{\epsilon}^{\sigma}(\hat{\mathbf{k}})
$$

and

$$
H^{\prime}{ }_{I}=\frac{e^{2}}{2 m}|\mathbf{A}|^{2} .
$$

If we rescale the electronic charge as $e \hookrightarrow \lambda e$ we find that

$$
H \hookrightarrow H_{S}+H_{R}+\lambda H_{I}+\lambda^{2} H_{I}^{\prime} .
$$

In the subsequent analysis we shall drop the term $\lambda^{2} H^{\prime}{ }_{I}$ and consider only

$$
H^{(\lambda)}=H_{S}+H_{R}+\lambda H_{I}
$$

It has been established rigorously that this does not effect the final result in the weak coupling limit. Now the interaction $H_{I}$ given by (120) has response terms described by the vectors $\theta_{j}^{\sigma}(\mathbf{k})=\frac{i e}{\hbar m} e^{-i \mathbf{k} \cdot \mathbf{r}} G_{j}^{\sigma}(\hat{\mathbf{k}}) \cdot \mathbf{p}$. We assume, as usual, that the unperturbed system Hamiltonian $H_{S}$ has a complete orthonormal set of of eigenstates $B$. In the case of the Hydrogen atom, this means that we consider only the bound states and ignore the effect of the ionized states. In general, $\mathcal{H}_{\mathcal{S}}$ can be decomposed into complementary subspaces generated by the discrete, the absolutely continuous and the singular parts of the spectrum of $H_{S}$. It is enough to prepare the system in the discrete spectrum subspace to apply our results. It is the standard approach in atomic physics to study only the behaviour of bound states anyway, so we are justified in this restriction.

We introduce the test-functions

$$
g_{\phi \phi^{\prime}}^{\sigma}(\mathbf{k})=\frac{i e}{\hbar m}\left\langle\phi\left|e^{-i \mathbf{k} \cdot \mathbf{r}} \mathbf{p}\right| \phi^{\prime}\right\rangle \cdot \mathbf{G}^{\sigma}(\hat{\mathbf{k}}) .
$$

The interaction $H_{I}$ can be expressed as

$$
\begin{gathered}
H_{I}=\sum_{\phi, \phi^{\prime} \in B} \sum_{\sigma=1,2} \int d^{3} k\left\{a_{\sigma}^{\dagger}(\mathbf{k}) g_{\phi \phi^{\prime}}^{\sigma}(\mathbf{k})-a_{\sigma}(\mathbf{k}) \bar{g}_{\phi \phi^{\prime}}^{\sigma}(\mathbf{k})\right\} \otimes T_{\phi \phi^{\prime}} \\
=\sum_{\phi, \phi^{\prime} \in B} \sum_{\sigma=1,2} \int d^{3} k\left\{T_{\phi \phi^{\prime}} \otimes a_{\sigma}^{\dagger}(\mathbf{k}) g_{\phi \phi^{\prime}}^{\sigma}(\mathbf{k})-T_{\phi^{\prime} \phi} \otimes a_{\sigma}(\mathbf{k}) \bar{g}_{\phi \phi^{\prime}}^{\sigma}(\mathbf{k})\right\} \\
=\sum_{\phi, \phi^{\prime} \in B}\left\{T_{\phi \phi^{\prime}} \otimes A^{\dagger}\left(g_{\phi \phi^{\prime}}\right)-T_{\phi \phi^{\prime}}^{\dagger} \otimes A\left(g_{\phi \phi^{\prime}}\right)\right\}
\end{gathered}
$$


where $g_{\phi \phi^{\prime}}=g_{\phi \phi^{\prime}}^{1} \oplus g_{\phi \phi^{\prime}}^{2} \in L^{2}\left(R^{3}\right) \oplus L^{2}\left(R^{3}\right)=\mathcal{H}_{R}^{1}$ and $A^{\sharp}$ are the creation/ annihilation operators on $\Gamma_{B}\left(\mathcal{H}_{R}^{1}\right)=$ $\otimes \Gamma_{B}\left(L^{2}(\mathbb{R})\right)$;

$$
\begin{aligned}
A^{\dagger}\left(f^{1} \oplus f^{2}\right) & =\sum_{\sigma=1,2} \int d^{3} k f^{\sigma}(\mathbf{k}) a_{\sigma}^{\dagger}(\mathbf{k}), \\
A\left(f^{1} \oplus f^{2}\right) & =\sum_{\sigma=1,2} \int d^{3} k \overline{f^{\sigma}}(\mathbf{k}) a_{\sigma}(\mathbf{k}) .
\end{aligned}
$$

Our choice of $\mathcal{H}_{R}^{1}$ above for one particle of the reservoir space is quite natural; namely it consists of wave-functions in the momentum representation with two transverse polarizations. The state of the reservoir is in our case determined by the covariance operator $Q$ which we shall now specify as that of a thermal field at inverse temperature $\beta\rangle 0$, that is

$$
\begin{gathered}
Q: \mathcal{H}_{R}^{1} \mapsto \mathcal{H}_{R}^{1}: h_{1} \oplus h_{2} \mapsto \tilde{h}_{1} \oplus \tilde{h}_{2} \\
\text { with } \tilde{h}_{\sigma}(\mathbf{k})=q(c|\mathbf{k}|) h_{\sigma}(\mathbf{k}),
\end{gathered}
$$

where $q(\omega)=\operatorname{coth} \frac{\beta \hbar \omega}{2}$. With $\omega_{\phi \phi^{\prime}}=\left(E_{\phi^{\prime}}-E_{\phi}\right) / \hbar \in F$, we define

$$
S_{t}^{\omega_{\phi \phi^{\prime}}}: \mathcal{H}_{R}^{1} \mapsto \mathcal{H}_{R}^{1}: h_{1} \oplus h_{2} \mapsto \tilde{h}_{1} \oplus \tilde{h}_{2}
$$

with

$$
\tilde{h}_{\sigma}(\mathbf{k})=e^{i\left(c|\mathbf{k}|-\omega_{\phi \phi^{\prime}}\right) t} h_{\sigma}(\mathbf{k}) .
$$

In this setup we have allowed for the most general coupling, that is where all the fundamental frequencies $F=\left\{\omega_{\phi \phi^{\prime}}\right.$ : $\left.\phi, \phi^{\prime} \in B\right\}$ are to be considered. This set is always degenerate in general; however it is important to consider two classes of degeneracy arising. The first is the secular class; these are the situations in which degeneracies always arise regardless of the spectrum $\left\{E_{\phi}: \phi \in B\right\}$ of $H_{S}$; they are the pairs $\left(\phi, \phi^{\prime}\right)$ and $\left(\psi, \psi^{\prime}\right)$ which have $\omega_{\phi \phi^{\prime}}=\omega_{\psi \psi^{\prime}}$ due to one of the following reasons

$$
\begin{gathered}
\text { 1. } \phi=\phi^{\prime}=\psi=\psi^{\prime}, \\
\text { 2. } \phi=\phi^{\prime}, \psi=\psi^{\prime} ;(\phi \neq \psi), \\
\text { 3. } \phi=\psi, \phi^{\prime}=\psi^{\prime} ;\left(\phi \neq \phi^{\prime}\right) .
\end{gathered}
$$

Any solution to the equation $\omega_{\phi \phi^{\prime}}=\omega_{\psi \psi^{\prime}}$, or equivalently $E_{\phi}-E_{\phi^{\prime}}=E_{\psi}-E_{\psi^{\prime}}$, not of the secular type shall be called an extraneous solution. The extraneous solutions are of course dependent on the spectrum of $H_{S}$. It is standard procedure in the physical literature to assume that such possibilities do not arise however this is a requirement on $H_{S}$ which cannot be made in many important examples. For a particle in a rectangular box, apart from the natural degeneracies arising if the ratios of the sides are rational, we also have to consider the fact that the contribution to the energy for the mode of vibration $n_{i}$ along the $i^{\text {th }}$-axis is proportional to $n_{i}^{2}$, this means solving the Diophantine equations for the harmonics

$$
n_{i}^{2}-m_{i}^{2}=n_{i}^{\prime 2}-m_{i}^{\prime 2}
$$

For the Hydrogen atom we have, apart from the spherical harmonical degeneracies, to consider the integer solutions to the Diophantine equations

$$
\frac{1}{n^{2}}-\frac{1}{m^{2}}=\frac{1}{n^{\prime 2}}-\frac{1}{m^{\prime 2}}
$$

for the principal atomic numbers. After simple manipulations this leads to the study of the intersection of the algebraic projective curve in $\mathbb{R}^{4}$;

$$
x_{1}^{2} x_{3}^{2} x_{4}^{2}-x_{2}^{2} x_{3}^{2} x_{4}^{2}-x_{1}^{2} x_{2}^{2} x_{4}^{2}+x_{1}^{2} x_{2}^{2} x_{3}^{2}=0
$$

with the lattice of positive integers. In the weak coupling limit we obtain the quantum stochastic differential equation

$$
d U_{t}=\left[\sum_{\omega \in F} \sum_{\phi, \phi^{\prime} \in B}^{\left(\omega_{\phi \phi^{\prime}}=\omega\right)}\left\{T_{\phi \phi^{\prime}} \otimes d B_{Q}^{\omega \dagger}\left(g_{\phi \phi^{\prime}}, t\right)+T_{\phi \phi^{\prime}}^{\dagger} \otimes d B_{Q}^{\omega}\left(g_{\phi \phi^{\prime}}, t\right)\right\}+Y d t\right] U_{t}
$$

where

$$
Y=\sum_{\omega \in F} \sum_{\phi, \phi^{\prime}, \psi, \psi^{\prime} \in B}^{\left(\omega_{\phi \phi^{\prime}}=\omega=\omega_{\psi \psi^{\prime}}\right)}\left[T_{\phi \phi^{\prime}}^{\dagger} T_{\psi \psi^{\prime}}\left(g_{\phi \phi^{\prime}} \mid g_{\psi \psi^{\prime}}\right)_{Q+}^{\omega-}+T_{\phi \phi^{\prime}} T_{\psi \psi^{\prime}}^{\dagger} \overline{\left(g_{\phi \phi^{\prime}} \mid g_{\psi \psi^{\prime}}\right)_{Q-}^{\omega-}}\right]
$$


with $U_{0}=1$. However using the fact that $T_{\phi \phi^{\prime}}^{\dagger} T_{\psi \psi^{\prime}}=\langle\phi, \psi\rangle T_{\phi^{\prime} \psi^{\prime}}$ etc., we may write $Y$ as

$$
Y=\sum_{\omega \in F} \sum_{\phi, \psi, \phi^{\prime} \in B}^{\left(\omega=\omega_{\phi \phi^{\prime}}=\omega_{\psi \phi^{\prime}}\right)}\left\{\left(g_{\phi^{\prime} \phi} \mid g_{\phi^{\prime} \psi}\right)_{Q+}^{(-\omega)-}+\overline{\left(g_{\phi \phi^{\prime}} \mid g_{\psi \phi^{\prime}}\right)_{Q-}^{\omega-}}\right\} T_{\phi \psi}
$$

In the summation we consider only $\phi$ and $\psi$ for which there exists a $\phi^{\prime}$ so that $\omega_{\phi \phi^{\prime}}=\omega_{\psi \phi^{\prime}}$, however this is equivalent to demanding that $\omega_{\phi \psi}=0$ as we always have the identity $\omega_{\phi \psi}=\omega_{\phi \phi^{\prime}}-\omega_{\psi \phi^{\prime}}$. Therefore $Y$ is a linear combination of terms $T_{\phi \psi}$ with $\omega_{\phi \psi}=0$ and this, in particular, implies that $Y$ commutes with $H_{S}$. It is natural in light of this to write $Y$ as

$$
Y=\sum_{\phi, \psi \in B}^{\left(\omega_{\phi \psi}=0\right)} y_{\phi \psi} T_{\phi \psi}=\sum_{\phi, \psi \in B}^{\left(E_{\phi}=E_{\psi}\right)} y_{\phi \psi} T_{\phi \psi}
$$

where

$$
y_{\phi \psi}=\sum_{\phi^{\prime} \in B}\left\{\left(g_{\phi^{\prime} \phi} \mid g_{\phi^{\prime} \psi}\right)_{Q+}^{\omega_{\phi^{\prime} \phi}-}+\overline{\left(g_{\phi \phi^{\prime}} \mid g_{\psi \phi^{\prime}}\right)_{Q-}^{\omega_{\phi \phi^{\prime}}-}}\right\} .
$$

Now the master equation associated with this problem is from (105)

$$
\frac{d s_{t}}{d t}=L_{0}^{*}\left(s_{t}\right)=-\left(Y s_{t}+s_{t} Y^{\dagger}\right)+\sum_{\omega \in F} \sum_{\phi, \phi^{\prime}, \psi, \psi^{\prime} \in B}^{\left(\omega=\omega_{\phi \phi^{\prime}}=\omega_{\psi \psi^{\prime}}\right)}\left[T_{\phi \phi^{\prime}}^{\dagger} s_{t} T_{\psi \psi^{\prime}}\left(g_{\phi \phi^{\prime}} \mid g_{\psi \psi^{\prime}}\right)_{Q+}^{\omega}+T_{\phi \phi^{\prime}} s_{t} T_{\psi \psi^{\prime}}^{\dagger}\left(g_{\psi \psi^{\prime}} \mid g_{\phi \phi^{\prime}}\right)_{Q-}^{\omega}\right]
$$

In order to find the general expression for $H_{S}^{\prime}$, we see that

$$
\begin{gathered}
y_{\phi \psi}=\sum_{\phi^{\prime} \in B} \int_{-\infty}^{0} d \tau\left\{\left\langle g_{\phi^{\prime} \phi}, S_{\tau}^{\omega_{\phi^{\prime} \phi}} \frac{Q+1}{2} g_{\phi^{\prime} \psi}\right\rangle+\overline{\left\langle g_{\phi \phi^{\prime}}, S_{\tau}^{\omega_{\phi \phi^{\prime}}} \frac{Q-1}{2} g_{\psi \phi^{\prime}}\right\rangle}\right\} \\
=\sum_{\phi^{\prime} \in B} \int_{-\infty}^{0} d \tau \sum_{\sigma=1,2} \int d^{3} k\left\{\overline{g_{\phi^{\prime} \phi}^{\sigma}}(\mathbf{k}) g_{\phi^{\prime} \psi}^{\sigma}(\mathbf{k}) e^{-i c \mid(\mathbf{k} \mid t} \frac{q(c|\mathbf{k}|)+1}{2}+g_{\phi \phi^{\prime}}^{\sigma}(\mathbf{k}) \overline{g_{\psi \phi^{\prime}}^{\sigma}}(\mathbf{k}) e^{i c|\mathbf{k}| t} \frac{q(c|\mathbf{k}|)-1}{2}\right\} e^{i \omega_{\phi^{\prime} \phi^{\prime}} t} .
\end{gathered}
$$

But using $\overline{g_{\phi \phi^{\prime}}^{\sigma}}(\mathbf{k})=-g_{\phi^{\prime} \phi}^{\sigma}(-\mathbf{k})$ we have

$$
\begin{aligned}
y_{\phi \psi}= & \sum_{\phi^{\prime} \in B} \int_{-\infty}^{0} d \tau \sum_{\sigma=1,2} \int d^{3} k g_{\phi \phi^{\prime}}^{\sigma}(\mathbf{k}) \overline{g_{\psi \phi^{\prime}}^{\sigma}}(\mathbf{k})\left\{e^{-i c|\mathbf{k}| t} \frac{q(c|\mathbf{k}|)+1}{2}+e^{i c|\mathbf{k}| t} \frac{q(c|\mathbf{k}|)-1}{2}\right\} e^{i \omega_{\phi^{\prime} \phi^{t}} t} \\
= & \frac{e^{2}}{\hbar^{2} m^{2}} \sum_{\phi^{\prime} \in B} \int_{-\infty}^{0} d \tau \sum_{\sigma=1,2} \int d^{3} k \sum_{j, j^{\prime}=1,2,3}\left\langle\phi\left|e^{-i \mathbf{k} \cdot \mathbf{r}} p_{j}\right| \phi^{\prime}\right\rangle\left\langle\phi^{\prime}\left|e^{i \mathbf{k} \cdot \mathbf{r}} p_{j^{\prime}}\right| \psi\right\rangle \\
& \times G_{j}^{\sigma}(\mathbf{k}) G_{j^{\prime}}^{\sigma}(\mathbf{k})\left\{e^{-i c|\mathbf{k}| t} \frac{q(c|\mathbf{k}|)+1}{2}+e^{i c|\mathbf{k}| t} \frac{q(c|\mathbf{k}|)-1}{2}\right\} e^{i \omega_{\phi^{\prime} \phi^{t}}} \\
= & \frac{e^{2}}{\hbar^{2} m^{2}} \sum_{\phi^{\prime} \in B} \int_{-\infty}^{0} d \tau \sum_{\sigma=1,2} \int d^{3} k \sum_{j, j^{\prime}=1,2,3}\left\langle\phi\left|e^{\frac{t}{i \hbar} E_{\phi}} e^{-i \mathbf{k} \cdot \mathbf{r}} p_{j} e^{\frac{-t}{i \hbar} H_{S}} e^{i \mathbf{k} \cdot \mathbf{r}} p_{j^{\prime}}\right| \psi\right\rangle \\
& \times G_{j}^{\sigma}(\mathbf{k}) G_{j^{\prime}}^{\sigma}(\mathbf{k})\left\{e^{-i c|\mathbf{k}| t} \frac{q(c|\mathbf{k}|)+1}{2}+e^{i c|\mathbf{k}| t} \frac{q(c|\mathbf{k}|)-1}{2}\right\} .
\end{aligned}
$$

We remark that the effect of the response term is as follows; from the commutation relations of $\mathbf{r}$ and $\mathbf{p}$ we have that

$$
e^{-i \mathbf{k} \cdot \mathbf{r}} e^{\frac{t}{i \hbar} H_{S}} e^{i \mathbf{k} \cdot \mathbf{r}}=e^{-i \mathbf{k} \cdot \mathbf{r}} \exp \frac{t}{i \hbar}\left(\frac{|\mathbf{p}|^{2}}{2 m}+V(r)\right) e^{i \mathbf{k} \cdot \mathbf{r}}=\exp \frac{t}{i \hbar}\left(\frac{|\mathbf{p}+\hbar \mathbf{k}|^{2}}{2 m}+V(r)\right)
$$

that is $H_{S}$ is replacd by $H_{S}+\frac{\hbar \mathbf{k} \cdot \mathbf{p}}{m}+\frac{\hbar^{2}|\mathbf{k}|^{2}}{2 m}$. Now the $\mathbf{k}$ dependence in the above expression prevents us from using the well-known isotropic identity

$$
\sum_{\sigma=1,2} \int_{|\mathbf{k}|=\omega / c} d^{2} \hat{k} G_{j}^{\sigma}(\mathbf{k}) G_{j^{\prime}}^{\sigma}(\mathbf{k})=\frac{1}{(2 \pi)^{3}} \frac{\hbar}{2 \epsilon_{0} \omega} \frac{8 \pi}{3} \delta_{j, j^{\prime}}
$$


to calculate $y_{\phi \psi}$ as in the dipole approximation. Note that the Lamb shift and the damping coefficients are effected by inclusion of the response terms.

The complex shift $Y_{\phi \phi}=\frac{1}{2} \Gamma_{\phi}+\frac{i}{\hbar} E_{\phi}^{\prime}$, giving the linewidth $\Gamma_{\phi}$ and energy shift $E_{\phi}^{\prime}$ for a state $\phi \in B$ can the be written as

$$
Y_{\phi \phi}=\frac{e^{2}}{2 i \hbar m^{2}} \sum_{\sigma=1,2} \int d^{3} k \sum_{j, j^{\prime}=1,2,3}\left\langle\phi\left|p_{j}\left[\frac{q(c|\mathbf{k}|)+1}{\mathcal{D}^{+}(\mathbf{k})-i 0^{+}}+\frac{q(c|\mathbf{k}|)-1}{\mathcal{D}^{-}(\mathbf{k})-i 0^{+}}\right] p_{j^{\prime}}\right| \phi\right\rangle G_{j}^{\sigma}(\mathbf{k}) G_{j^{\prime}}^{\sigma}(\mathbf{k}),
$$

where the denominators in the above expression are

$$
\mathcal{D}^{ \pm}(\mathbf{k})=H_{S}+\frac{\hbar \mathbf{k} \cdot \mathbf{p}}{m}+\frac{\hbar^{2}|\mathbf{k}|^{2}}{2 m} \pm \hbar c|\mathbf{k}|-E_{\phi} .
$$

This expression has been derived in [20] for the zero temperature case; see however [21].

APPENDIX. A: The Traditional Derivation of the Master Equation.

For the sake of comparison we give the standard arguments used in the derivation of the master equation. This section follows closely the development of Louisell [2]. The interaction is taken to be of the form

$$
H_{I}=\sum_{j} D_{j} \otimes F_{j}
$$

where $D_{j}$ and $F_{j}$ act nontrivially on the system and reservoir spaces respectively. We assume that $D_{j}$ evolves harmonically in time under the free evolution with frequency $\omega_{j}$ We assume that at time $t$ the system and reservoir are uncoupled, that is the density operator $\rho(t)$ at time zero factors as

$$
\rho(0)=\rho_{0}^{(S)} \otimes \rho^{(R)} .
$$

No subscript is required for $\rho^{(R)}$ as we assume that it is invariant under the free-evolution (in particular this is true for the choice of a thermal state $\left.\rho^{(R)}=e^{-\beta\left(H_{R}^{1}-\mu\right)} / \operatorname{Tr} e^{-\beta\left(H_{R}^{1}-\mu\right)}\right)$. We define the reduced system state at time $t$ in the interaction dynamics to be the density operator

$$
s_{t}=\operatorname{Tr}_{\mathcal{H}_{R}}\left\{U_{t}^{(\lambda)}\left(\rho_{0}^{(S)} \otimes \rho^{(R)}\right) U_{t}^{(\lambda) \dagger}\right\} .
$$

The iterated series expansion of $s_{t}$, truncated to second order, is;

$$
s_{t}=s_{0}+\frac{1}{i \hbar} \int_{0}^{t} d t_{1} \operatorname{Tr}_{\mathcal{H}_{R}}\left[v_{t_{1}}^{(0)}\left(H_{I}\right), s_{0} \otimes \rho^{(R)}\right]+\frac{1}{(i \hbar)^{2}} \int_{0}^{t} d t_{1} \int_{0}^{t_{1}} d t_{2} \operatorname{Tr}_{\mathcal{H}_{R}}\left[v_{t_{1}}^{(0)}\left(H_{I}\right),\left[v_{t_{2}}^{(0)}\left(H_{I}\right), s_{0} \otimes \rho^{(R)}\right]\right]
$$

where we have set $\lambda=1$. Substituting in for the potential $H_{I}$ we find

$$
\begin{aligned}
s_{t}= & s_{0}+\sum_{j} \int_{0}^{t} d t_{1}\left\langle v_{t_{1}}^{(0)}\left(F_{j}\right)\right\rangle_{R} e^{-i \omega_{j} t_{1}}\left[D_{j}, s_{0}\right] \\
& +\sum_{j, k} \int_{0}^{t} d t_{1} \int_{0}^{t_{1}} d t_{2} e^{-i\left(\omega_{j} t_{1}+\omega_{k} t_{2}\right)}\left\{\left[D_{j} D_{k} s_{0}-D_{k} s_{0} D_{j}\right]\left\langle v_{t_{1}}^{(0)}\left(F_{j}\right) v_{t_{2}}^{(0)}\left(F_{k}\right)\right\rangle_{R}-\left[D_{j} s_{0} D_{k}-s_{0} D_{k} D_{j}\right]\left\langle v_{t_{2}}^{(0)}\left(F_{k}\right) v_{t_{1}}^{(0)}\left(F_{j}\right)\right\rangle_{R}\right\}
\end{aligned}
$$

where $\langle.\rangle_{R}=\operatorname{Tr}_{\mathcal{H}_{R}}\left[\rho^{(R)}.\right]$. Due to the invariance of the reservoir fields under the free evolution we have that

$$
\begin{aligned}
\left\langle v_{t}^{(0)}\left(F_{j}\right)\right\rangle_{R} & =\left\langle F_{j}\right\rangle_{R} ; \\
\left\langle v_{t}^{(0)}\left(F_{j}\right) v_{s}^{(0)}\left(F_{k}\right)\right\rangle_{R} & =\left\langle v_{t-s}^{(0)}\left(F_{j}\right) F_{k}\right\rangle_{R} .
\end{aligned}
$$

Therefore, if we let $\tau=t_{1}-t_{2}, y=t_{2}$ then we obtain

$$
\begin{aligned}
s_{t}= & s_{0}+\sum_{j}\left\langle F_{j}\right\rangle_{R}\left[D_{j}, s_{0}\right] \int_{0}^{t} e^{-i \omega_{j} y} d y \\
& +\sum_{j, k} \int_{0}^{t} d y e^{-i\left(\omega_{j}+\omega_{k}\right) y} \int_{0}^{t-y} d \tau e^{-i \omega_{j} \tau}\left\{\left[D_{j} D_{k} s_{0}-D_{k} s_{0} D_{j}\right]\left\langle v_{\tau}^{(0)}\left(F_{j}\right) F_{k}\right\rangle_{R}-\left[D_{j} s_{0} D_{k}-s_{0} D_{k} D_{j}\right]\left\langle F_{k} v_{\tau}^{(0)}\left(F_{j}\right)\right\rangle_{R}\right\} .
\end{aligned}
$$


The approximation procedure is based on the following four steps; step I. One postulates that the contributions coming from the sum of all terms higher that second order in the iterated series are negligible. step II. One postulates a finite autocorrelation time $\tau_{c}$ such that

$$
\left\langle v_{\tau}^{(0)}\left(F_{j}\right) F_{k}\right\rangle_{R}=0=\left\langle F_{j} v_{\tau}^{(0)}\left(F_{k}\right)\right\rangle_{R},
$$

whenever $|\tau|\rangle \tau_{c}$. Thus for $\left.\left.t\right\rangle\right\rangle \tau_{c}$ one may replace the upper limit of the $\tau$-integral by $+\infty$. This gives

$$
\begin{gathered}
s_{t}=s_{0}+\sum_{j}\left\langle F_{j}\right\rangle_{R}\left[D_{j}, s_{0}\right] I^{t}\left(\omega_{j}\right) \\
+\sum_{j, k}\left\{\left[D_{j} D_{k} s_{0}-D_{k} s_{0} D_{j}\right] w_{j, k}^{+}-\left[D_{j} s_{0} D_{k}-s_{0} D_{k} D_{j}\right] w_{k, j}^{-}\right\} I^{t}\left(\omega_{j}+\omega_{k}\right),
\end{gathered}
$$

where

$$
\begin{aligned}
& w_{j, k}^{+}=\int_{0}^{\infty} e^{-i \omega_{j} \tau}\left\langle v_{\tau}^{(0)}\left(F_{j}\right) F_{k}\right\rangle_{R} d \tau \\
& w_{k, j}^{-}=\int_{0}^{\infty} e^{-i \omega_{j} \tau}\left\langle F_{k} v_{\tau}^{(0)}\left(F_{j}\right)\right\rangle_{R} d \tau
\end{aligned}
$$

and

$$
I^{t}(\omega)=\int_{0}^{t} e^{-i \omega y} d y
$$

Step III. For $t$ large with respect to $\tau_{c}$ one makes the replacement

$$
I^{t}(\omega) \hookrightarrow t \delta(\omega) .
$$

Step IV. One postulates that the formulas deduced under the previous assumptions when $t$ is large with respect to $\tau_{c}$ hold also in the limit $t \rightarrow 0$; this gives

$$
\begin{gathered}
\left.\frac{d s}{d t}\right|_{0}=\lim _{t \rightarrow 0} \frac{s_{t}-s_{0}}{t} \\
=\sum_{j}^{\omega_{j}=0}\left[D_{j}, s_{0}\right]\left\langle F_{j}\right\rangle_{R}+\sum_{j, k}^{\omega_{j}+\omega_{k}=0}\left\{\left[D_{j} D_{k} s_{0}-D_{k} s_{0} D_{j}\right] w_{j, k}^{+}-\left[D_{j} s_{0} D_{k}-s_{0} D_{k} D_{j}\right] w_{k, j}^{-}\right\} .
\end{gathered}
$$

The assumptions leading to this equation have a decidedly ad hoc nature, especially those introduced in steps III and IV. The replacement for $I^{t}$, put in by hand, in step III is precisely what is needed to allow the limit to be taken easily. It is instructive to calculate explicitly the master equation in a particular case. We consider as reservoir a free Bose gas at inverse temperature $\beta$ and fugacity $z=e^{\beta \mu}$. This can be described by the quasi-free state $\varphi_{Q}$, on $L^{2}\left(\mathbb{R}^{n}\right)$ for example, characterized by

$$
\left\langle A^{\dagger}(f) A(g)\right\rangle_{R} \equiv \varphi_{Q}\left(A^{\dagger}(f) A(g)\right)=\left\langle f, \frac{Q-1}{2} g\right\rangle,
$$

where

$$
Q=\frac{1+z e^{-\beta H_{R}^{1}}}{1-z e^{-\beta H_{R}^{1}}}=\operatorname{coth} \frac{\beta}{2}\left(H_{R}^{1}-\mu\right) .
$$

We may take $H_{R}^{1}$ to be for instance $-\Delta$.

We may write the interaction $H_{I}$ of (31) in the form we are considering with the notations

$$
H_{I}=i \hbar \sum_{j}\left\{D_{j} \otimes A^{\dagger}\left(g_{j}\right)-h . c .\right\} \equiv i \hbar \sum_{(j, \alpha)} D_{(j, \alpha)} \otimes F_{(j, \alpha)},
$$

where we have a summation also over an index $\alpha \in\{0,1\}$ with the notations

$$
D_{(j, 0)}=D_{j}, D_{(j, 1)}=-D_{j}^{\dagger} ; F_{(j, 0)}=A^{\dagger}\left(g_{j}\right), F_{(j, 1)}=A\left(g_{j}\right) ;
$$

and consequently

$$
\omega_{(j, 0)}=\omega_{j} ; \omega_{(j, 1)}=-\omega_{j}
$$


We then have

$$
\begin{aligned}
w_{(j 0),(k 1)}^{+} & =\int_{0}^{\infty} d \tau e^{-i \omega_{j} \tau}\left\langle v_{\tau}^{(0)}\left(A^{\dagger}\left(g_{j}\right)\right) A\left(g_{k}\right)\right\rangle_{R}=\int_{0}^{\infty} d \tau \varphi_{Q}\left(A^{\dagger}\left(S_{\tau}^{\omega_{j}} g_{j}\right) A\left(g_{k}\right)\right) \\
& =\int_{0}^{\infty} d \tau\left\langle S_{\tau}^{\omega_{j}} g_{j}, \frac{Q-1}{2} g_{k}\right\rangle={\overline{\left(g_{j} \mid g_{k}\right)_{Q-}}}_{Q j_{j}-},
\end{aligned}
$$

Similarly, using the CCR, we find

$$
\begin{aligned}
w_{(j 1),(k 0)}^{+}=\int_{0}^{\infty} d \tau e^{i \omega_{j} \tau}\left\langle v_{\tau}^{(0)}\left(A\left(g_{j}\right)\right) A^{\dagger}\left(g_{k}\right)\right\rangle_{R}=\left(g_{j} \mid g_{k}\right)_{Q+}^{\omega_{j}-}, \\
w_{(k 0),(j 1)}^{-}=\left(g_{j} \mid g_{k}\right)_{Q-}^{\omega_{j}-}, \\
w_{(j 1),(k 0)}^{-}={\overline{\left(g_{j} \mid g_{k}\right)}}_{Q+}^{\omega_{j}-},
\end{aligned}
$$

while $w_{(j \epsilon),\left(j^{\prime} \epsilon^{\prime}\right)}^{ \pm}=0$ if $\epsilon=\epsilon^{\prime}$ as we have $\langle A(f) A(g)\rangle_{R}=0=\left\langle A^{\dagger}(f) A^{\dagger}(g)\right\rangle_{R}$. We note that $\left\langle F_{(j, \alpha)}\right\rangle_{R}=0$ in all cases. The master equation then reads

$$
\begin{aligned}
\left.\frac{d s}{d t}\right|_{0}= & \sum_{j, k ; \alpha, \alpha^{\prime}}{ }^{(-1)^{\alpha} \omega_{j}+(-1)^{\alpha^{\prime}} \omega_{k}=0}\left\{\left[D_{j, \alpha} D_{k, \alpha^{\prime}} s_{0}-D_{k, \alpha^{\prime}} s_{0} D_{j \alpha}\right] w_{(j \alpha),\left(k \alpha^{\prime}\right)}^{+}\right. \\
& \left.-\left[D_{j, \alpha} s_{0} D_{k, \alpha^{\prime}}-s_{0} D_{k, \alpha^{\prime}} D_{j, \alpha}\right] w_{\left(k \alpha^{\prime}\right),(j \alpha)}^{-}\right\} \\
= & -\sum_{j, k}^{\omega_{j}-\omega_{k}=0}\left\{\left[D_{j} D_{k}^{\dagger} s_{0}-D_{k}^{\dagger} s_{0} D_{j}\right] w_{(j 0),(k 1)}^{+}+\left[D_{j}^{\dagger} D_{k} s_{0}-D_{k} s_{0} D_{j}^{\dagger}\right] w_{(j 1),(k 0)}^{+}\right. \\
& \left.-\left[D_{j}^{\dagger} s_{0} D_{k}-s_{0} D_{k} D_{j}^{\dagger}\right] w_{(k 0),(j 1)}^{-}-\left[D_{j} s_{0} D_{k}^{\dagger}-s_{0} D_{k}^{\dagger} D_{j}\right] w_{(k 1),(j 0)}^{-}\right\},
\end{aligned}
$$

or writing in our notations (and employing the relabeling in terms of the frequency degeneracies as in (31))

$$
\begin{aligned}
\left.\frac{d s}{d t}\right|_{0}= & -\sum_{\omega \in F} \sum_{j, k=1}^{N(\omega)}\left\{\left[D_{j}^{\omega} D_{k}^{\omega \dagger} s_{0}-D_{k}^{\omega \dagger} s_{0} D_{j}^{\omega}\right] \overline{\left(g_{j}^{\omega} \mid g_{k}^{\omega}\right)_{Q-}^{\omega-}}\right. \\
& +\left[D_{j}^{\omega \dagger} D_{k}^{\omega} s_{0}-D_{k}^{\omega} s_{0} D_{j}^{\omega \dagger}\right]\left(g_{j}^{\omega} \mid g_{k}^{\omega}\right)_{Q+}^{\omega-} \\
& \left.-\left[D_{j}^{\omega \dagger} s_{0} D_{k}^{\omega}-s_{0} D_{k}^{\omega} D_{j}^{\omega \dagger}\right]\left(g_{j}^{\omega} \mid g_{k}^{\omega}\right)_{Q-}^{\omega-}-\left[D_{j}^{\omega} s_{0} D_{k}^{\omega \dagger}-s_{0} D_{k}^{\omega \dagger} D_{j}^{\omega}\right] \overline{\left(g_{j}^{\omega} \mid g_{k}^{\omega}\right)_{Q+}^{\omega-}}\right\} .
\end{aligned}
$$

But this is exactly $\left.\frac{d s_{t}}{d t}\right|_{0}=L_{0}^{*}\left(s_{0}\right)$, where $L_{0}^{*}$ is given as before.

APPENDIX B: The Convergence of the Collective Processes to the Noise

\section{Processes}

The mathematical theory behind the weak coupling limit developed in [1] and subsequent papers is the following. We estimate the behaviour as $\lambda \rightarrow 0$ of matrix elements of $U_{t / \lambda^{2}}^{(\lambda)}$ with respect to collective coherent vectors, that is vectors of the form $B_{t^{(1)} / \lambda^{2}}^{(\omega, \lambda)}\left(f^{(1)}\right) \ldots B_{t^{(n)} / \lambda^{2}}^{(\omega, \lambda)}\left(f^{(n)}\right) \Psi_{R}$. This involves substituting $v_{t}\left(H_{I}\right)$ into the series expansion for $U_{t / \lambda^{2}}^{(\lambda)}$ and examining each of the terms arising. The deep analysis of [1] shows that each term upon normal ordering leads to two classes of terms: relevant ones (type I) and negligible ones (type II). The type I terms are exactly those put into normal order by commuting time consecutive pairs of reservoir variables, the type II terms account for all others. Following this resummation it is shown that the type II terms give vanishing contribution in the limit $\lambda \rightarrow 0$ while the explicit limit for the type I terms is calculated; uniform convergence is established, the main technical device used here is one of various generalizations of the Pule inequality [3].

The independence of the noise processes for different frequencies follows from the next two lemmas.

Lemma 1: for each $\omega \in F$ let $f_{\omega}, f_{\omega}^{\prime} \in K_{\omega}$ and $S_{\omega}, T_{\omega}, S_{\omega}^{\prime}, T_{\omega}^{\prime} \in R$ then

$$
\begin{aligned}
\lim _{\lambda \rightarrow 0} & \left\langle\lambda \int_{S_{\omega} / \lambda^{2}}^{T_{\omega} / \lambda^{2}} S_{u}^{\omega} f_{\omega} d u, \lambda \int_{S^{\prime}{ }_{\omega} / \lambda^{2}}^{T^{\prime}{ }_{\omega} / \lambda^{2}} S_{v}^{\omega} f^{\prime}{ }_{\omega} d v\right\rangle \\
& =\sum_{\omega \in F}\left\langle\chi_{\left[S_{\omega}, T_{\omega}\right]}, \chi_{\left[S^{\prime}{ }_{\omega}, T^{\prime}{ }_{\omega}\right]}\right\rangle_{L^{2}(\mathbb{R})}\left(f_{\omega} \mid f^{\prime}{ }_{\omega}\right)_{\omega} \\
& =\left(\oplus_{\omega \in F}\left(\chi_{\left[S_{\omega}, T_{\omega}\right]} \otimes f_{\omega}\right) \mid \oplus_{\omega^{\prime} \in F}\left(\chi_{\left[S^{\prime}{ }^{\prime}, T^{\prime}{ }^{\prime}\right]} \otimes f^{\prime}{ }_{\omega^{\prime}}\right) .\right.
\end{aligned}
$$


Proof. the left hand side of (B.1) can be written as a sum over $\omega, \omega^{\prime} \in F$ of terms

$$
\lim _{\lambda \rightarrow 0} \int_{S_{\omega}}^{T_{\omega}} d u \int_{\left(S^{\prime}{ }^{\prime}-u\right) / \lambda^{2}}^{\left(T^{\prime}{ }_{\omega^{\prime}}-u\right) / \lambda^{2}} d u^{\prime}\left\langle f_{\omega}, S_{u^{\prime}}^{\omega} f^{\prime}{ }_{\omega^{\prime}}\right\rangle e^{i\left(\omega-\omega^{\prime}\right) u / \lambda^{2}}
$$

by the Riemann-Lesbegue Lemma the terms $\omega \neq \omega^{\prime}$ vanish while the $\omega=\omega^{\prime}$ terms converge by inspection to

$$
\left.\left\langle\chi_{\left[S_{\omega}, T_{\omega}\right]}, \chi_{\left[S^{\prime}{ }_{\omega}, T^{\prime} \omega\right.}\right]\right\rangle_{L^{2}(\mathbb{R})}\left(f_{\omega} \mid f^{\prime}{ }_{\omega}\right)_{\omega}
$$

Lemma 2: for $n \in N$ let $f_{\omega}^{(k)} \in K_{\omega}, x_{\omega}^{(k)} \in R, S_{\omega}^{(k)}\left\langle T_{\omega}^{(k)}\right.$, for $1 \leq k \leq n$ and each $\omega \in F$, then

$$
\lim _{\lambda \rightarrow 0}\left\langle\Phi_{Q}^{F}, B_{Q}^{F \dagger}\left(\sum_{\omega \in F} x_{\omega}^{(1)} \lambda \int_{S_{\omega}^{(1)}}^{T_{\omega}^{(1)}} S_{u_{1}}^{\omega} f_{\omega}^{(1)} d u_{1}\right) \ldots B_{Q}^{F \dagger}\left(\sum_{\omega \in F} x_{\omega}^{(n)} \lambda \int_{S_{\omega}^{(n)}}^{T_{\omega}^{(n)}} S_{u_{n}}^{\omega} f_{\omega}^{(n)} d u_{n}\right) \Phi_{Q}^{F}\right\rangle
$$

exists uniformly for the $x$ 's and $[S, T]$ 's in a bounded set of $R$ and is equal to

$$
\left\langle\Phi_{R}^{Q}, W\left(\oplus_{\omega \in F}\left(x_{\omega}^{(1)} \chi_{\left[S_{\omega}^{(1)}, T_{\omega}^{(1)}\right]} \otimes f_{\omega}^{(1)}\right)\right) \ldots W\left(\oplus_{\omega \in F}\left(x_{\omega}^{(n)} \chi_{\left[S_{\omega}^{(n)}, T_{\omega}^{(n)}\right]} \otimes f_{\omega}^{(n)}\right)\right) \Phi_{R}^{Q}\right\rangle .
$$

For a proof see [10].

\section{APPENDIX C: The Quantum Stochastic Differential Equation for $U_{t}$.}

For convenience we shall consider only one coupling frequency $\omega$ so that

$$
H_{I} \equiv i \hbar \sum_{j}\left(D_{j} \otimes A^{\dagger}\left(g_{j}\right)\right)
$$

and we have dropped the superscript $\omega$ from the operators. Also we shall consider only the Fock (vacuum) case $Q=1$. We define $G_{\lambda}(t)$ by

$$
\left\langle\psi, G_{\lambda}(t)\right\rangle=\operatorname{Tr}\left\{|\phi\rangle\left\langle\psi\left|\otimes B_{T^{\prime} / \lambda^{2}}^{(\omega, \lambda) \dagger}\left(f^{\prime}\right)\right| \Psi_{R}\right\rangle\left\langle\Psi_{R}\right| B_{T / \lambda^{2}}^{(\omega, \lambda)}(f) U_{t / \lambda^{2}}^{(\lambda)}\right\},
$$

where $\psi, \phi \in \mathcal{H}_{S}$ and the second inner product is meant on $\mathcal{H}_{S} \otimes \Gamma_{B}\left(L^{2}\left(\mathbb{R}, K_{\omega}\right)\right)$ : we know that the $\operatorname{limit}_{\lim } \rightarrow 0\left\langle\psi, G_{\lambda}(t)\right\rangle$ exists and equals

$$
\left\langle\psi \otimes B_{Q}^{\omega \dagger}(f, T) \Phi_{Q}^{\omega}, U_{t} \phi \otimes B_{Q}^{\omega \dagger}\left(f^{\prime}, T^{\prime}\right) \Phi_{Q}^{\omega}\right\rangle .
$$

It is easy to show that this limit has the form $\langle\psi, G(t)\rangle$ where $: t \mapsto G(t) \in \mathcal{H}_{S}$ is weakly differentiable. In order to obtain a differential equation for $G(t)$ we note that for fixed $\lambda$ one has

$$
\begin{aligned}
\frac{d}{d t}\left\langle\psi, G_{\lambda}(t)\right\rangle & =\operatorname{Tr}\left\{|\phi\rangle\left\langle\psi\left|\otimes B_{T^{\prime} / \lambda^{2}}^{(\omega, \lambda) \dagger}\left(f^{\prime}\right)\right| \Psi_{R}\right\rangle\left\langle\Psi_{R}\right| B_{T / \lambda^{2}}^{(\omega, \lambda)}(f) \frac{1}{\lambda} \sum_{j}\left(D_{j} \otimes A^{\dagger}\left(S_{t / \lambda^{2}}^{\omega} g_{j}\right)-D_{j}^{\dagger} \otimes A\left(S_{t / \lambda^{2}}^{\omega} g_{j}\right)\right) U_{t / \lambda^{2}}^{(\lambda)}\right\} \\
& =\Gamma_{\lambda}+\Xi_{\lambda} ;
\end{aligned}
$$

where

$$
\begin{aligned}
& \Gamma_{\lambda}=\frac{1}{\lambda} \operatorname{Tr}\left\{|\phi\rangle\left\langle\psi\left|\otimes B_{T^{\prime} / \lambda^{2}}^{(\omega, \lambda) \dagger}\left(f^{\prime}\right)\right| \Psi_{R}\right\rangle\left\langle\Psi_{R}\right| B_{T / \lambda^{2}}^{(\omega, \lambda)}(f) \sum_{j} D_{j} \otimes A^{\dagger}\left(S_{t / \lambda^{2}}^{\omega} g_{j}\right) U_{t / \lambda^{2}}^{(\lambda)}\right\}, \\
& \Xi_{\lambda}=-\frac{1}{\lambda} \operatorname{Tr}\left\{|\phi\rangle\left\langle\psi\left|\otimes B_{T^{\prime} / \lambda^{2}}^{(\omega, \lambda) \dagger}\left(f^{\prime}\right)\right| \Psi_{R}\right\rangle\left\langle\Psi_{R}\right| B_{T / \lambda^{2}}^{(\omega, \lambda)}(f) \sum_{j} D_{j}^{\dagger} \otimes A\left(S_{t / \lambda^{2}}^{\omega} g_{j}\right) U_{t / \lambda^{2}}^{(\lambda)} .\right.
\end{aligned}
$$


Now

$$
\begin{aligned}
\Gamma_{\lambda} & =\sum_{j} \frac{1}{\lambda} \lambda \int_{0}^{T / \lambda^{2}}\left\langle S_{u}^{\omega} f, S_{t / \lambda^{2}}^{\omega} g_{j}\right\rangle d u\left\langle D_{j}^{\dagger} \psi, G_{\lambda}(t)\right\rangle \\
& =\sum_{j} \frac{1}{\lambda} \lambda \int_{(-t) / \lambda^{2}}^{(T-t) / \lambda^{2}}\left\langle S_{v}^{\omega} f, g_{j}\right\rangle d v\left\langle D_{j}^{\dagger} \psi, G_{\lambda}(t)\right\rangle
\end{aligned}
$$

where we made the substitution $u-t / \lambda^{2}=v$. We see that for bounded $D$ this converges as $\lambda \rightarrow 0$ a.e. to

$$
\sum_{j} \chi_{[S, T]}\left(f \mid g_{j}\right)_{\omega}\left\langle D_{j}^{\dagger} \psi, G_{\lambda}(t)\right\rangle \text {. }
$$

Next of all the term $\Xi_{\lambda}$ must be reordered as follows

$$
\begin{aligned}
\Xi_{\lambda}= & -\frac{1}{\lambda} \sum_{j} \operatorname{Tr}\left\{|\phi\rangle\left\langle\psi\left|\otimes B_{T^{\prime} / \lambda^{2}}^{(\omega, \lambda) \dagger}\left(f^{\prime}\right)\right| \Psi_{R}\right\rangle\left\langle\Psi_{R}\right| B_{T / \lambda^{2}}^{(\omega, \lambda)}(f)\left(D_{j}^{\dagger} \otimes 1\right) U_{t / \lambda}^{(\lambda)}\left(1 \otimes A\left(S_{t / \lambda^{2}}^{\omega}\right)\right)\right\} \\
& -\frac{1}{\lambda} \sum_{j} \operatorname{Tr}\left\{|\phi\rangle\left\langle\psi\left|\otimes B_{T^{\prime} / \lambda^{2}}^{(\omega, \lambda) \dagger}\left(f^{\prime}\right)\right| \Psi_{R}\right\rangle\left\langle\Psi_{R}\right| B_{T / \lambda^{2}}^{(\omega, \lambda)}(f)\left(D_{j}^{\dagger} \otimes 1\right)\left[\left(1 \otimes A\left(S_{t / \lambda^{2}}^{\omega}\right)\right), U_{t / \lambda}^{(\lambda)}\right]\right\} \\
= & \Xi_{\lambda}^{a}+\Xi_{\lambda}^{b} .
\end{aligned}
$$

In a fashion similar to the calculation of $\Gamma_{\lambda}$, one easily arrives at

$$
\lim _{\lambda \rightarrow 0} \Xi_{\lambda}^{a}=-\sum_{j} \chi_{\left[0, T^{\prime}\right]}\left(g_{j} \mid f^{\prime}\right)_{\omega}\left\langle D_{j}^{\dagger} \psi, G_{\lambda}(t)\right\rangle \text {, a.e. }
$$

To evaluate the limit of $\Xi_{\lambda}^{b}$ we note that

$$
\begin{aligned}
{\left[\left(1 \otimes A\left(S_{t / \lambda^{2}}^{\omega} g_{j}\right)\right), U_{t / \lambda^{2}}^{(\lambda)}\right]=} & \sum_{n=1}^{\infty}\left(\frac{\lambda}{i \hbar}\right)^{n} \int_{0}^{t / \lambda^{2}} d t_{1} \int_{0}^{t_{1}} d t_{2} \ldots \int_{0}^{t_{n-1}} d t_{n}\left[\left(1 \otimes A\left(S_{t / \lambda^{2}}^{\omega} g_{j}\right)\right), H_{I}\left(t_{1}\right) \ldots H_{I}\left(t_{n}\right)\right] \\
= & \sum_{n=1}^{\infty}\left(\frac{\lambda}{i \hbar}\right)^{n} \int_{0}^{t / \lambda^{2}} d t_{1} \int_{0}^{t_{1}} d t_{2} \ldots \int_{0}^{t_{n-1}} d t_{n}\left\{\left[\left(1 \otimes A\left(S_{t / \lambda^{2}}^{\omega} g_{j}\right)\right), H_{I}\left(t_{1}\right)\right] H_{I}\left(t_{2}\right) \ldots H_{I}\left(t_{n}\right)\right. \\
& +H_{I}\left(t_{1}\right)\left[\left(1 \otimes A\left(S_{t / \lambda^{2}} g_{j}\right), H_{I}\left(t_{2}\right) \ldots H_{I}\left(t_{n}\right)\right]\right\} .
\end{aligned}
$$

It can be shown that in the limit $\lambda \rightarrow 0$ only the commutator involving $H_{I}\left(t_{1}\right)$ contributes. Hence

$$
\begin{gathered}
\lim _{\lambda \rightarrow 0} \Xi_{\lambda}^{b}=-\lim _{\lambda \rightarrow 0} \sum_{n=1}^{\infty}\left(\frac{\lambda}{i \hbar}\right)^{n-1} \int_{0}^{t / \lambda^{2}} d t_{1} \int_{0}^{t_{1}} d t_{2} \ldots \int_{0}^{t_{n-1}} d t_{n} \\
\sum_{j, k}\left\langle S_{t / \lambda^{2}}^{\omega} g_{j}, S_{t_{1}}^{\omega} g_{k}\right\rangle \operatorname{Tr}\left\{|\phi\rangle\left\langle\psi\left|\otimes B_{T^{\prime} / \lambda^{2}}^{(\omega, \lambda) \dagger}\left(f^{\prime}\right)\right| \Psi_{R}\right\rangle\left\langle\Psi_{R}\right| B_{T / \lambda^{2}}^{(\omega, \lambda)}(f) D_{j}^{\dagger} D_{k} H_{I}\left(t_{2}\right) \ldots H_{I}\left(t_{n}\right)\right\},
\end{gathered}
$$

but this is the same as

$$
\begin{aligned}
& -\lim _{\lambda \rightarrow 0} \frac{1}{\lambda^{2}} \sum_{j, k} \int_{0}^{t} d s\left\langle S_{t / \lambda^{2}}^{\omega} g_{j}, S_{s / \lambda^{2}}^{\omega} g_{k}\right\rangle D_{j}^{\dagger} D_{k} U_{s / \lambda^{2}}^{(\lambda)} \\
= & -\lim _{\lambda \rightarrow 0} \frac{1}{\lambda^{2}} \sum_{j, k} \int_{0}^{t} d s\left\langle S_{t / \lambda^{2}}^{\omega} g_{j}, S_{s / \lambda^{2}}^{\omega} g_{k}\right\rangle\left\langle D_{k}^{\dagger} D_{j} \psi, G_{\lambda}(t)\right\rangle=-\sum_{j, k}\left(g_{j} \mid g_{k}\right)^{\omega-}\left\langle\psi, D_{j}^{\dagger} D_{k} G(t)\right\rangle,
\end{aligned}
$$

where we have used a technical lemma (6.3) of [1].

We now have

$$
\begin{aligned}
\langle\psi, G(t)\rangle= & \lim _{\lambda \rightarrow 0}\left\langle\psi, G_{\lambda}(t)\right\rangle=\langle\psi, G(0)\rangle+\lim _{\lambda \rightarrow 0} \int_{0}^{t}\left(\Gamma_{\lambda}(s)+\Xi_{\lambda}(s)\right) d s \\
= & \langle\psi, G(0)\rangle+\int_{0}^{t} d s\left\{\sum_{j} \chi_{[S, T]}(s)\left(f \mid g_{j}\right)_{\omega}\left\langle D_{j}^{\dagger} \psi, G(s)\right\rangle\right. \\
& \left.-\sum_{j} \chi_{\left[S^{\prime}, T^{\prime}\right]}(s)\left(g_{j} \mid f\right)^{\omega}\left\langle\psi, D_{j} G(s)\right\rangle-\sum_{j, k}\left(g_{j} \mid g_{k}\right)^{\omega-}\left\langle\psi, D_{j}^{\dagger} D_{k} G(s)\right\rangle\right\} .
\end{aligned}
$$


Here we have written $(g \mid f)^{\omega-}$ for $(g \mid f)_{Q+}^{\omega-}$ when $Q=1$.

The quantum stochastic differential equation corresponding to this integral equation is

$$
d U_{t}=\left\{\sum_{j}\left(D_{j} \otimes d B_{Q}^{\omega \dagger}\left(g_{j}, t\right)-D_{j}^{\dagger} \otimes d B_{Q}^{\omega}\left(g_{j}, t\right)\right)-\sum_{j, k}\left(g_{j} \mid g_{k}\right)^{\omega-} D_{j}^{\dagger} D_{k} d t\right\} U_{t} .
$$

The generalization to $Q>1$ and several coupling frequencies $\omega$ is now obvious.

\section{References}

[1] Accardi,L. ,Frigerio,A. and Lu, Y.G.:The weak coupling limit as a quantum functional central limit. Comm.Math.Phys. 131,537-570(1990).2cm

[2] Louisell,W. :Quantum statistical properties of radiation. John Wiley and Sons.(1973).

[3] Pulé,J.V. :The Bloch equations. Comm.Math.Phys. 38,241-256(1974)

[4] Davies,E.B. :Markovian master equation. Comm.Math.Phys. 39,91-110(1974)

[5] Haken,H. :Laser Theory. Springer(1984)

[6] Lax,M. :Phys.Rev. 145,111-129(1965)

[7] von Waldenfels,W. :Ito solution of the linear quantum stochastic differential equation describing light emission and absorption. SLNM 1055 (ed Accardi, Frigerio, Gorini)

[8] Accardi,L. ,Frigerio,A. and Lu, Y.G.:The weak coupling limit (II): The Langevin equation and finite temperature case. Preprint Volterra Centro No.13 (1989)

[9] Accardi,L. ,Frigerio,A. and Lu, Y.G.:The weak coupling limit for Fermions. Preprint Volterra Centro No.12 (1989)

[10] Accardi,L. ,Frigerio,A. and Lu, Y.G.:The weak coupling limit without rotating wave approximation. Preprint Volterra Centro No.23 (1990)

[11] Accardi,L. ,Frigerio,A. and Lu, Y.G.:Unified Approach to the Quantum Master and Langevin Equations. Preprint Volterra Centro No.69 (1991)

[12] Accardi,L. and Lu, Y.G.:On the weak coupling limit for quantum electrodynamics. Prob. Meth. in Math. Phys. (ed F.Guerra, M.I. Loffredo, C. Marchro) World Scientific (1992) pgs 16-22.

[13] Hudson,R.L., Parthasarathy,K.R. :Quantum Ito's formula and stochastic evolutions. Comm.Math. Phys. 131,537$570(1990)$

[14] Parthasarathy,K.R.: An introduction to Quantum Statistical Calculus. Monographs in Mathematics. Birkhauser (1992)

[15] Kittel,G. ,Quantum theory of solids, John Wiley and Sons(1963)

[16] N.N.Bogolubov and N.N.Bogolubov Jnr. Some applications of the Polaron Theory. World Scientific Lecture Notes in Mathematics, vol 4 (1992)

[17] Sewell, G.L., Quantum Theory of Collective Phenomena, Monographs on the physics and chemiistry of materials, Oxford Science Publications. (1986) $.2 \mathrm{~cm}$

[18] Collett, M.J., Gardiner, C.W.: Phys.Rev A 1386 (1984)

[19] Messiah, A.: Quantum Mechanics. Vol II, North Holland Publishing Company. (1961)

[20] Au, C-K. and Feinberg,G.: Phys. Rev. A 9 ,1974 (1974) and 12,1772 (1975)

[21] Ford,G.W. von Waldenfels,W.: Radiative Energy Shifts for a Nonrelativistic Atom. 